\title{
Subgame maxmin strategies in zero-sum stochastic games with tolerance levels
}

Citation for published version (APA):

Flesch, J., Herings, P. J-J., Maes, J., \& Predtetchinski, A. (2018). Subgame maxmin strategies in zerosum stochastic games with tolerance levels. Maastricht University, Graduate School of Business and Economics. GSBE Research Memoranda No. 020 https://doi.org/10.26481/umagsb.2018020

Document status and date:

Published: 14/08/2018

DOI:

10.26481/umagsb.2018020

Document Version:

Publisher's PDF, also known as Version of record

\section{Please check the document version of this publication:}

- A submitted manuscript is the version of the article upon submission and before peer-review. There can be important differences between the submitted version and the official published version of record.

People interested in the research are advised to contact the author for the final version of the publication, or visit the DOI to the publisher's website.

- The final author version and the galley proof are versions of the publication after peer review.

- The final published version features the final layout of the paper including the volume, issue and page numbers.

Link to publication

\footnotetext{
General rights rights.

- You may freely distribute the URL identifying the publication in the public portal. please follow below link for the End User Agreement:

www.umlib.nl/taverne-license

Take down policy

If you believe that this document breaches copyright please contact us at:

repository@maastrichtuniversity.nl

providing details and we will investigate your claim.
}

Copyright and moral rights for the publications made accessible in the public portal are retained by the authors and/or other copyright owners and it is a condition of accessing publications that users recognise and abide by the legal requirements associated with these

- Users may download and print one copy of any publication from the public portal for the purpose of private study or research.

- You may not further distribute the material or use it for any profit-making activity or commercial gain

If the publication is distributed under the terms of Article $25 \mathrm{fa}$ of the Dutch Copyright Act, indicated by the "Taverne" license above, 
János Flesch,

P. Jean-Jacques Herings, Jasmine Maes,

Arkadi Predtetchinski

Subgame maxmin strategies in zero-sum stochastic games with tolerance levels

$\mathrm{RM} / 18 / 020$

\section{GSBE}

Maastricht University School of Business and Economics

Graduate School of Business and Economics

P.O Box 616

NL- 6200 MD Maastricht

The Netherlands 


\title{
Subgame maxmin strategies in zero-sum stochastic games with tolerance levels
}

\author{
János Flesch ${ }^{*}$ P. Jean-Jacques Herings ${ }^{\dagger}$ Jasmine Maes ${ }^{\ddagger}$ Arkadi Predtetchinski ${ }^{\S}$
}

July 18, 2018

\begin{abstract}
We study subgame $\phi$-maxmin strategies in two-player zero-sum stochastic games with finite action spaces and a countable state space. Here $\phi$ denotes the tolerance function, a function which assigns a non-negative tolerated error level to every subgame. Subgame $\phi$ maxmin strategies are strategies of the maximizing player that guarantee the lower value in every subgame within the subgame-dependent tolerance level as given by $\phi$. First, we provide necessary and sufficient conditions for a strategy to be a subgame $\phi$-maxmin strategy. As a special case we obtain a characterization for subgame maxmin strategies, i.e. strategies that exactly guarantee the lower value at every subgame. Secondly, we present sufficient conditions for the existence of a subgame $\phi$-maxmin strategy. Finally, we show the possibly surprising result that the existence of subgame $\phi$-maxmin strategies for every positive tolerance function $\phi$ is equivalent to the existence of a subgame maxmin strategy.
\end{abstract}

Keywords: Stochastic games, zero-sum games, subgame $\phi$-maxmin strategies.

${ }^{*}$ Department of Quantitative Economics, Maastricht University, P.O. Box 616, 6200 MD Maastricht, The Netherlands. E-Mail: J.Flesch@maastrichtuniversity.nl

${ }^{\dagger}$ Department of Economics, Maastricht University, P.O. Box 616, 6200 MD Maastricht, The Netherlands. EMail: P.Herings@maastrichtuniversity.nl

$\ddagger$ Department of Economics, Maastricht University, P.O. Box 616, 6200 MD Maastricht, The Netherlands. EMail: Jasmine.Maes@maastrichtuniversity.nl

$\S$ Department of Economics, Maastricht University, P.O. Box 616, 6200 MD Maastricht, The Netherlands. EMail: A.Predtetchinski@maastrichtuniversity.nl 


\section{Introduction}

Two-player zero-sum stochastic games model the repeated interaction between two agents with opposite objectives. The environment in which the interaction takes place is fully characterized by a state variable. The transition from one state variable to the next one is influenced by both players as well as an element of chance. Throughout the paper we take the perspective of the maximizing player. We are interested in strategies of the maximizing player that guarantee the lower value at every subgame and call such strategies subgame maxmin strategies. Under the assumptions as made in the paper, the value may not exist, which explains why we consider the lower value instead.

As the name subgame maxmin strategy suggests, this concept is closely related to the concept of a subgame perfect equilibrium as defined in Selten (1965). In two-player zero-sum games where the value exists, for conditions see Maitra and Sudderth (1998) and Martin (1998), the notions of a subgame maxmin strategy and a subgame minmax strategy coincide with the notion of a subgame optimal strategy. Moreover, in such games a strategy profile is a subgame perfect equilibrium if and only if it consists of a pair of subgame optimal strategies.

As illustrated by the Big Match, a game introduced in Gillette (1957) and analyzed in Blackwell and Ferguson (1968), even if the value exists, it is not guaranteed that optimal strategies exist, so a fortiori, subgame optimal strategies and subgame perfect equilibria may not exist. A large part of the literature therefore focuses on so-called subgame perfect $\epsilon$ equilibria as defined in Radner (1980). This equilibrium concept is more permissive than a subgame perfect equilibrium and consists of a strategy pair such that every player obtains the value at each history up to a fixed error term of $\epsilon / 2$.

Instead of having a fixed error term at each subgame, we allow the error term to vary across different subgames. This error term is expressed as a function $\phi$ of the histories and is called the tolerance function. The central topic of this paper is the concept of a subgame $\phi$-maxmin strategy. This is a strategy of the maximizing player that guarantees the lower value at every subgame within the allowed tolerance level. Intuitively, a subgame $\phi$-maxmin strategy performs sufficiently well across all subgames. This type of strategy is related to the concept of $\phi$-tolerance equilibrium as defined in Flesch and Predtetchinski (2016). Indeed, if the value exists, then a strategy profile in which both players use a subgame $(\phi / 2)$-optimal strategy is a $\phi$-tolerance equilibrium.

One motivation for letting the tolerance level vary across subgames is given by Mailath, Postlewaite, and Samuelson (2005) when introducing the concept of a contemporaneous perfect $\epsilon$-equilibrium. The authors focus on games in which the payoff function of the players is given by the discounted sum of periodic rewards. Due to this discounting, there exists a period after which the maximal total discounted reward a player can receive is smaller than $\epsilon$. If the allowed tolerance level $\epsilon$ is fixed across all subgames, any strategy will be an $\epsilon$-maxmin strategy of a subgame in such a period. Therefore, the concept of subgame $\epsilon$-maxmin strategy does not impose any restrictions on the actions chosen at a very distant future. The issue here is that it would be more intuitive to discount not only the reward but also the allowed tolerance level.

Additional motivation for letting the tolerance level vary across subgames stems from the 
fact that the notion of what is considered sufficiently good might be relative. For instance, Tversky and Kahneman (1985) observe that people evaluate decisions with respect to a reference level. They find that significantly more people were willing to exert extra effort to save $\$ 5$ on a $\$ 15$ purchase than to save $\$ 5$ on a $\$ 125$ purchase. To apply this to the context of zero-sum games, consider the following game to which we will refer as the high stakes-low stakes game. In the first stage of the game, a chance move determines whether the player will engage in the high stakes or the low stakes variant of this game. The high stakes and the low stakes games are identical in terms of possible strategies. The only difference is that the payoffs in the high stakes game are a thousand fold the payoffs in the low stakes game. Furthermore, assume that in the high stakes subgame the payoff of player 1 ranges between 0 and 2000 and the value is 1000 , while in the low stakes subgame the payoff of player 1 ranges between 0 and 2 and the value is 1 . Players that evaluate decisions with respect to a reference level, may label a strategy which guarantees a payoff of 999 in the high stakes game as sufficiently good. However, in the low stakes game, 0 corresponds to the minimum payoff. Allowing the tolerance level to vary across subgames can therefore be used to accommodate such behavioral effects into the model of zero-sum stochastic games.

Another case where history dependent tolerance levels are natural is the following. In situations that commonly occur, a player may use a familiar strategy irrespective of the scale of the payoffs. To understand this better, imagine a player who is highly experienced in playing a certain zero-sum game. He has a trusted strategy which guarantees him the value of this game within some error. Now consider the high stakes-low stakes game again. The player might well use the trusted strategy in both the low stakes and the high stakes subgame. Therefore the error related to this strategy will be relative with respect to the value of the respective subgame.

Finally, in the class of stochastic games as identified in Flesch, Thuijsman and Vrieze (1998), the only way to obtain $\epsilon$-optimality is to use strategies that are called improving. Improving strategies are closely related to subgame $\phi$-maxmin strategies such that the tolerance level in some subgames is smaller than the tolerance level at the root.

With respect to the concept of subgame $\phi$-maxmin strategies, this paper attempts to provide answers to the following three fundamental questions:

1. What are the necessary and sufficient conditions for a strategy to be a subgame $\phi$ maxmin strategy?

2. For positive tolerance functions $\phi$, when does a subgame $\phi$-maxmin strategy exist?

3. When does a subgame maxmin strategy exist?

The first question concerning the necessary and sufficient conditions for subgame $\phi$ maxmin strategies is answered in Section 4. As a special case of these necessary and sufficient conditions, we obtain a characterization of subgame maxmin strategies. For the special class of positive and negative stochastic games, a related characterization of subgame maxmin strategies was obtained by Flesch, Predtetchinski and Sudderth (2018).

The necessary and sufficient conditions for strategies to be subgame $\phi$-maxmin can be split into a local condition and an equalizing condition. Informally, the local condition states that the lower value one expects to get in the next subgame should always be at least the 
lower value of the current subgame. The equalizing condition requires that, for every strategy of the other player, a subgame $\phi$-maxmin strategy almost surely results in a play with an eventually good enough payoff, where eventually good enough means being at least the lower value in very deep subgames up to the allowed tolerance level.

The second and third question regarding the existence of subgame $\phi$-maxmin strategies are examined in Sections 5 and 6. In Section 5 we consider positive tolerance functions. The existence of subgame maxmin strategies is discussed in Section 6. The existence and construction of such strategies is important as they can serve as punishment strategies in multi-player games. This is illustrated in the paper of Mashiah-Yaakovi (2015).

We prove that for a positive tolerance function $\phi$, a subgame $\phi$-maxmin strategy exists if every play is either a point of upper semicontinuity of the payoff function or if the sequence of tolerance levels which occur along the play has a positive lower bound. We give a constructive proof of this statement using the sufficient conditions for a strategy to be subgame $\phi$-maxmin.

A special case of our theorem, where the sequence of tolerance levels which occur along the play always has a positive lower bound, has been studied in Mashiah-Yaakovi (2015). In Proposition 11 of that paper, the existence of a subgame $\epsilon$-optimal strategy in a twoplayer zero-sum stochastic game with Borel measurable payoff functions, finite action sets, and a countable state space has been shown. A subgame $\epsilon$-optimal strategy corresponds to a constant tolerance function that is everywhere equal to $\epsilon$.

Our main result in Section 6 states that the existence of a subgame $\phi$-maxmin strategy for every positive tolerance function $\phi$ is equivalent to the existence of a subgame maxmin strategy. For upper semi-continuous payoff functions, our theorem in Section 5 guarantees the existence of a subgame $\phi$-maxmin strategy for every positive tolerance function $\phi$, so it follows that a subgame maxmin strategy exists if the payoff function is upper semi-continuous. The latter conclusion is related to a result in Laraki, Maitra and Sudderth (2013).

The connection between existence of subgame $\phi$-maxmin strategies for every positive tolerance function $\phi$ and the existence of subgame maxmin strategies is not only useful to further understand the results obtained by Laraki, Maitra and Sudderth (2013) but also highlights an important and surprising difference between subgame $\phi$-maxmin strategies and the closely related concept of subgame $\epsilon$-maxmin strategies. Indeed, the existence of a subgame $\epsilon$-maxmin strategy for every $\epsilon>0$ does not imply the existence of a subgame maxmin strategy.

The rest of the paper is structured as follows. In Section 2 we formulate the model setup and in Section 3 we formally define the main concepts. Then in Section 4 we discuss the necessary and sufficient conditions for a strategy to be a subgame $\phi$-maxmin strategy and give a characterization for subgame maxmin strategies. We continue in Section 5 by providing sufficient conditions to guarantee the existence of a subgame $\phi$-maxmin strategy. Then in Section 6 we explain why the existence of subgame $\phi$-maxmin strategies for every positive tolerance function $\phi$ is equivalent to existence of a subgame maxmin strategy. Finally, in Section 7 we discuss the importance of the assumptions we made and whether they might be relaxed. 


\section{Two-player zero-sum stochastic games}

We consider a two-player zero-sum stochastic game with finitely many actions and countably many states. The payoff function is required to be bounded and universally measurable. The model encompasses all two-player zero-sum games with perfect information and stochastic moves.

Actions, states, and histories: The action sets of players 1 and 2 are given by the finite sets $\mathcal{A}$ and $\mathcal{B}$, respectively. The state space is given by the countable set $\mathcal{X}$. Let $x_{0}$ denote the initial state and define the set $\mathcal{Z}=\mathcal{A} \times \mathcal{B} \times \mathcal{X}$. The game consists of an infinite sequence of one-shot games. At the initial state $x_{0}$, the one-shot game $G\left(x_{0}\right)$ is played as follows: Players 1 and 2 simultaneously select an action from their respective action sets, denoted by $a_{1}$ and $b_{1}$, respectively. Then the next state $x_{1}$ is selected according to the transition probability $q\left(\cdot \mid x_{0}, a_{1}, b_{1}\right)$. At the new state $x_{1}$, this process repeats itself and both players play the one-shot game $G\left(x_{1}\right)$ by selecting actions $a_{2}$ and $b_{2}$ from their respective action sets. The next state $x_{2}$ is selected according to the transition probability $q\left(\cdot \mid x_{0}, a_{1}, b_{1}, x_{1}, a_{2}, b_{2}\right)$. This goes on indefinitely and creates a play $p=\left(x_{0}, a_{1}, b_{1}, x_{1}, a_{2}, b_{2}, \cdots\right)$. Note that the transition probability can depend on the entire history.

For every $t \in \mathbb{N}=\{0,1,2, \ldots\}$, let $\mathcal{H}^{t}=\left\{x_{0}\right\} \times \mathcal{Z}^{t}$ denote the set of all histories that are generated after $t$ one-shot games. The set $\mathcal{H}^{0}$ consists of the single element $x_{0}$. For $t \geq 1$, elements of $\mathcal{H}^{t}$ are of the form $\left(x_{0}, a_{1}, b_{1}, \ldots, a_{t}, b_{t}, x_{t}\right)$. Let $\mathcal{H}=\cup_{t \in \mathbb{N}} \mathcal{H}^{t}$ denote the set of all histories. For all $h \in \mathcal{H}$, let $\|h\|=\left\|\left(x_{0}, a_{1}, b_{1}, \ldots, a_{t}, b_{t}, x_{t}\right)\right\|=t$ denote the number of one-shot games that occurred during the history $h$. We refer to $\|h\|$ as the length of the history $h$. For all $t \leq\|h\|$, the restriction of the history $h$ to the first $t$ one-shot games is denoted by $h_{\mid t}=\left(x_{0}, a_{1}, b_{1}, \ldots, a_{t}, b_{t}, x_{t}\right)$. We write $h^{\prime} \preceq h$ if there exists $t \leq\|h\|$ such that $h_{\mid t}=h^{\prime}$, so if the history $h$ extends the history $h^{\prime}$.

The space of plays: Define $\mathcal{P}=\left\{x_{0}\right\} \times \mathcal{Z}^{\mathbb{N}}$ to be the set of plays. Elements of $\mathcal{P}$ are of the form $p=\left(x_{0}, a_{1}, b_{1}, x_{1}, a_{2}, b_{2}, \cdots\right)$. For $t \in \mathbb{N}$, let $p_{\mid t}$ denote the prefix of $p$ of length $t$ : that is $p_{\mid 0}=x_{0}$ and $p_{\mid t}=\left(x_{0}, a_{1}, b_{1}, \ldots, a_{t}, b_{t}, x_{t}\right)$ for $t \geq 1$. We write $h \prec p$ if a history $h$ is a prefix of $p$. For every $h \in \mathcal{H}$, let $\mathcal{P}(h)=\{p \in \mathcal{P} \mid h \prec p\}$ denote the cylinder set consisting of all plays which extend history $h$.

We endow $\mathcal{Z}$ with the discrete topology and $\mathcal{P}$ with the product topology. The collection of all cylinder sets is a basis for the product topology on $\mathcal{P}$.

For $t \in \mathbb{N}$, let $\mathcal{F}^{t}$ be the sigma-algebra generated by the collection of cylinder sets $\{\mathcal{P}(h) \mid$ $\left.h \in \mathcal{H}^{t}\right\}$. Each set in $\mathcal{F}^{t}$ can be written as the union of sets in $\left\{\mathcal{P}(h) \mid h \in \mathcal{H}^{t}\right\}$. Let $\mathcal{F}^{\infty}$ be the sigma-algebra generated by $\cup_{t \in \mathbb{N}} \mathcal{F}^{t}$. This is exactly the Borel sigma-algebra generated by the product topology on $\mathcal{P}$. The sigma-algebra of universally measurable subsets of $\mathcal{P}$ is denoted by $\mathcal{F}$. Elements of $\mathcal{F}$ are sets that belong to the completion of each Borel probability measure on $\mathcal{P}$. For details of the definition of the sigma-algebra $\mathcal{F}$, the reader is referred to Appendix A. It holds that $\mathcal{F}^{t} \subseteq \mathcal{F}^{t+1} \subseteq \cdots \subseteq \mathcal{F}^{\infty} \subseteq \mathcal{F}$. The set of plays $\mathcal{P}$ together with the universally measurable sigma-algebra $\mathcal{F}$ determines a measurable space $(\mathcal{P}, \mathcal{F})$. A stochastic variable is a universally measurable function from $\mathcal{P}$ to $\mathbb{R}$.

Strategies: Let $\Delta(\mathcal{A})$ denote the set of probability measures over the action set of player 1 
and $\Delta(\mathcal{B})$ the set of probability measures over the action set of player 2. A behavioral strategy for player 1 is a function $\sigma: \mathcal{H} \rightarrow \Delta(\mathcal{A})$. Hence, at each history player 1 chooses a mixed action. A pure strategy for player 1 is a function that assigns to every history an action, with a minor abuse of notation, $\sigma: \mathcal{H} \rightarrow \mathcal{A}$. Similarly, one can define a behavioral and a pure strategy $\tau$ for player 2. Let $\mathcal{S}_{1}$ and $\mathcal{S}_{2}$ denote the sets of behavioral strategies of players 1 and 2 , respectively.

It follows from the Ionescu Tulcea extension theorem that every history $h \in \mathcal{H}$ and strategy profile $(\sigma, \tau) \in \mathcal{S}_{1} \times \mathcal{S}_{2}$ determine a probability measure $\mathbb{P}_{h, \sigma, \tau}$ on the measurable space

$\left(\mathcal{P}(h), \mathcal{F}_{\mathcal{P}(h)}^{\infty}\right)$, where $\mathcal{F}_{\mathcal{P}(h)}^{\infty}$ denotes the Borel sigma-algebra over the set of plays extending the history $h$. The measure $\mathbb{P}_{h, \sigma, \tau}$ can be extended to the measurable space $\left(\mathcal{P}, \mathcal{F}^{\infty}\right)$ in the obvious way. Taking the completion of the probability space $\left(\mathcal{P}, \mathcal{F}^{\infty}, \mathbb{P}_{h, \sigma, \tau}\right)$ yields the probability space $\left(\mathcal{P}, \mathcal{F}, \mathbb{P}_{h, \sigma, \tau}^{C}\right)$. With a minor abuse of notation, we will omit the superscript $C$ and write $\mathbb{P}_{h, \sigma, \tau}$ in the remainder of this paper.

Payoff function: We assume that the payoff function $u: \mathcal{P} \rightarrow \mathbb{R}$ of player 1 is bounded and universally measurable. We also assume the game to be zero-sum. The payoff function of player 2 is therefore given by $-u$. We denote the game as described above by $\Gamma_{x_{0}}(u)$. Throughout the paper we take the point of view of player 1. This is without loss of generality, since the situation of Player 2 in the game $\Gamma_{x_{0}}(u)$ is identical to that of Player 1 in the game $\Gamma_{x_{0}}(-u)$.

The expected payoff of player 1 corresponding to strategy profile $(\sigma, \tau) \in \mathcal{S}_{1} \times \mathcal{S}_{2}$ at history $h \in \mathcal{H}$ is given by $\mathbb{E}_{h, \sigma, \tau}[u]$, where the expectation is taken with respect to the probability measure $\mathbb{P}_{h, \sigma, \tau}$. The expected payoff of player 1 at the history $x_{0}$ is denoted by $\mathbb{E}_{\sigma, \tau}[u]$.

Unlike two-player zero-sum stochastic games with Borel measurable payoff functions, twoplayer zero-sum stochastic games with universally measurable payoff functions do not necessarily have a value, formally defined in Section 3. The core idea of this paper, the construction and existence of strategies that perform sufficiently well in every subgame, is independent of the problem of the existence of a value. The reader unfamiliar with universally measurable payoff functions may therefore imagine Borel measurable payoff functions throughout the paper.

\section{$3 \quad$ Subgame $\phi$-maxmin strategies}

For every game $\Gamma_{x_{0}}(u)$, for every history $h \in \mathcal{H}$, we define the lower value $\underline{v}(h)$ and the upper value $\bar{v}(h)$ by

$$
\begin{aligned}
& \underline{v}(h)=\sup _{\sigma \in \mathcal{S}_{1}} \inf _{\tau \in \mathcal{S}_{2}} \mathbb{E}_{h, \sigma, \tau}[u], \\
& \bar{v}(h)=\inf _{\tau \in \mathcal{S}_{2}} \sup _{\sigma \in \mathcal{S}_{1}} \mathbb{E}_{h, \sigma, \tau}[u] .
\end{aligned}
$$

Because the payoff function $u$ is assumed to be bounded, we have that $\underline{v}(h), \bar{v}(h) \in \mathbb{R}$. Therefore, the lower and upper value exist in every subgame of $\Gamma_{x_{0}}(u)$. Furthermore, we have that $\underline{v}(h) \leq \bar{v}(h)$. Whenever $\underline{v}(h)=\bar{v}(h)$ we say that the subgame at history $h$ has a value and we denote it by $v(h)$. The lower value, the upper value, and the value at the initial state 


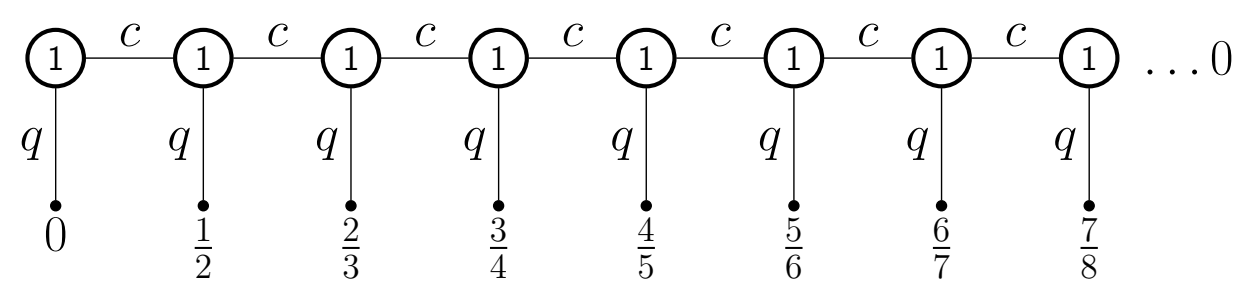

Figure 1: Characterization of $\phi$ such that pure subgame $\phi$-maxmin strategies exist.

$x_{0}$ are denoted by $\underline{v}, \bar{v}$, and $v$, respectively. If $u$ is Borel measurable, then the value exists by Maitra and Sudderth (1998) and Martin (1998). Since we do not assume $u$ to be Borel measurable, we present our results in terms of the lower value.

Even if the value exists, player 1 may not have a strategy that guarantees the value in each subgame and the literature has therefore studied subgame $\epsilon$-optimal strategies. These are strategies which guarantee the value in each subgame up to an allowed error term of $\epsilon$. If the payoff function is bounded and Borel measurable, it has been shown by MashiahYaakovi (2015) that for each $\epsilon>0$ player 1 has a subgame $\epsilon$-optimal strategy. The concept of a subgame $\epsilon$-optimal strategy has a constant error term $\epsilon$ across all subgames. However, as argued in the introduction, there are instances in which it is more natural to consider a variable error term. Therefore, instead of considering a constant error term, we follow Flesch and Predtetchinski (2016), who allow the error term to vary across histories in their investigation of the $\phi$-tolerance equilibrium. This leads us to the study of subgame $\phi$-maxmin strategies, where $\phi: \mathcal{H} \rightarrow[0, \infty)$ is a tolerance function assigning an allowed tolerance level to each history.

Definition 3.1. Let $\phi: \mathcal{H} \rightarrow[0, \infty)$ be a tolerance function. A strategy $\sigma \in \mathcal{S}_{1}$ is a subgame $\phi$-maxmin strategy in the game $\Gamma_{x_{0}}(u)$ if for every history $h \in \mathcal{H}$ it holds that

$$
\forall \tau \in \mathcal{S}_{2}, \mathbb{E}_{h, \sigma, \tau}[u] \geq \underline{v}(h)-\phi(h) .
$$

In case $\phi$ is identically equal to zero, we omit it from the notation, and simply refer to a subgame maxmin strategy.

A subgame $\phi$-maxmin strategy guarantees at each history $h$ of the game the lower value up to the tolerance level $\phi(h)$. If the tolerance function is such that, for some $\epsilon \geq 0$, for every $h \in \mathcal{H}, \phi(h)=\epsilon$, then we refer to the strategy as a subgame $\epsilon$-maxmin strategy. If the value exists, then the notion of subgame $\epsilon$-maxmin strategy coincides with the notion of subgame $\epsilon$-optimal strategy.

The following example illustrates that even for a strictly positive tolerance function $\phi$ player 1 may have no subgame $\phi$-maxmin strategies. Interestingly, however, player 1 has a subgame $\epsilon$-maxmin strategy for every positive $\epsilon>0$.

Example 3.2. The decision problem depicted in Figure 1 corresponds to a two-player zerosum stochastic game in which the state space is trivial and the second player is a dummy player. Whenever the state space or action sets are degenerate, the corresponding states and actions are omitted from the notation in examples. The set of actions of player 1 is $\mathcal{A}=\{c, q\}$, where $c$ stands for continue and $q$ for quit. The game stops as soon as player 1 chooses to 
quit. If player 1 decides to quit at period $t$, then his payoff is $t /(t+1)$. If player 1 never quits, his payoff is 0 .

In this game, player 1 has a subgame $\epsilon$-maxmin strategy for every positive $\epsilon>0$. For example, the strategy which quits whenever quitting leads to a payoff of at least $1-\epsilon$.

We now turn to the existence of a subgame $\phi$-maxmin strategy. Clearly, there exist no subgame maxmin strategy. As we will see later, Theorem 6.1 then implies that there is some strictly positive tolerance function $\phi$ for which there does not exist a subgame $\phi$-maxmin strategy. Intuitively, such a tolerance function forces player 1 to continue with such a large probability that the total probability of never quitting becomes nearly one.

In the remainder of this example we focus on pure strategies and we provide a characterization of tolerance functions $\phi$ for which there is a pure subgame $\phi$-maxmin strategy.

ClAIM: There exists a pure subgame $\phi$-maxmin strategy if and only if

1. for every $t \in \mathbb{N}, \phi\left(c^{t}\right)>0$,

2. for infinitely many $t \in \mathbb{N}, \phi^{\prime}\left(c^{t}\right)=\min _{n \leq t} \phi\left(c^{n}\right) \geq \frac{1}{t+1}$.

Proof: For every $t \in \mathbb{N}$, the value $v\left(c^{t}\right)$ exists and is equal to 1 . Hence any pure subgame $\phi$-maxmin strategy $\sigma$ has the property that, for every $t \in \mathbb{N}, u\left(\pi\left(\sigma, c^{t}\right)\right) \geq 1-\phi\left(c^{t}\right)$, where $\pi\left(\sigma, c^{t}\right)$ denotes the play induced from history $c^{t}$ when using strategy $\sigma$.

$\Rightarrow$ Because a payoff of exactly 1 can never be reached it is clear that $\phi\left(c^{t}\right)>0$ for every $t \in \mathbb{N}$.

Let $\sigma$ be a pure subgame $\phi$-maxmin strategy. We distinguish three cases.

CASE 1: For every $t \in \mathbb{N}, \sigma\left(c^{t}\right)=c$. For every $t \in \mathbb{N}$ it holds that $u\left(\pi\left(\sigma, c^{t}\right)\right)=u\left(c^{\infty}\right)=0$. Because $\sigma$ is a subgame $\phi$-maxmin strategy, we find that, for every $t \in \mathbb{N}, \phi\left(c^{t}\right) \geq 1$, so $\phi^{\prime}\left(c^{t}\right)=\min _{n \leq t} \phi\left(c^{n}\right) \geq 1 \geq 1 /(t+1)$.

CASE 2: The number of $t \in \mathbb{N}$ such that $\sigma\left(c^{t}\right)=q$ is positive and finite. Consider the increasing sequence of times $\left(t_{k}\right)_{k=0, \ldots, k^{\prime}}$ at which $\sigma\left(c^{t_{k}}\right)=q$. For $t \in\left\{0, \ldots, t_{0}\right\}$, we have that

$$
u\left(\pi\left(\sigma, c^{t}\right)\right)=u\left(c^{t_{0}} q\right)=\frac{t_{0}}{t_{0}+1},
$$

so $\phi\left(c^{t}\right) \geq 1 /\left(t_{0}+1\right)$ since $\sigma$ is a subgame $\phi$-maxmin strategy. We find that

$$
\phi^{\prime}\left(t_{0}\right)=\min _{t \in\left\{0, \ldots, t_{0}\right\}} \phi(t) \geq \frac{1}{t_{0}+1} .
$$

We argue next that if, for some $k \geq 1, t_{k-1}$ and $t_{k}$ are quitting times, then $\min _{t \in\left\{t_{k-1}+1, \ldots, t_{k}\right\}} \phi\left(c^{t}\right) \geq$ $1 /\left(t_{k}+1\right)$. Indeed, for $t \in\left\{t_{k-1}+1, \ldots, t_{k}\right\}$ we have that

$$
u\left(\pi\left(\sigma, c^{t}\right)\right)=u\left(c^{t_{k}} q\right)=\frac{t_{k}}{t_{k}+1},
$$

so $\phi\left(c^{t}\right) \geq 1 /\left(t_{k}+1\right)$ since $\sigma$ is a subgame $\phi$-maxmin strategy. Using induction, we find for $k=1, \ldots, k^{\prime}$ that

$$
\phi^{\prime}\left(c^{t_{k}}\right) \geq \min \left\{\phi^{\prime}\left(c^{t_{k-1}}\right), \frac{1}{t_{k}+1}\right\} \geq \frac{1}{t_{k}+1} .
$$


For every $t>t^{k^{\prime}}$ it holds that $\sigma\left(c^{t}\right)=c$ and $u\left(\pi\left(\sigma, c^{t}\right)\right)=u\left(c^{\infty}\right)=0$. For every $t>t^{k^{\prime}}$, since $\sigma$ is a subgame $\phi$-maxmin strategy, we have $\phi\left(c^{t}\right) \geq 1$, so

$$
\phi^{\prime}\left(c^{t}\right) \geq \min \left\{\phi^{\prime}\left(c^{t^{k^{\prime}}}\right), 1\right\} \geq \frac{1}{t^{k^{\prime}}+1}>\frac{1}{t+1},
$$

which concludes this case.

CASE 3: The number of $t \in \mathbb{N}$ such that $\sigma\left(c^{t}\right)=q$ is infinite. Consider the increasing sequence of times $\left(t_{k}\right)_{k \in \mathbb{N}}$ at which $\sigma\left(c^{t_{k}}\right)=q$. As in Case 2 it can be shown that for every $k \in \mathbb{N}$ it holds that $\phi^{\prime}\left(c^{t_{k}}\right) \geq 1 /\left(t_{k}+1\right)$.

$\Leftarrow$ Let the strategy $\sigma$ be defined as follows. For $t \in \mathbb{N}$,

$$
\sigma\left(c^{t}\right)= \begin{cases}q, & \text { if } \phi^{\prime}\left(c^{t}\right) \geq \frac{1}{t+1}, \\ c, & \text { otherwise. }\end{cases}
$$

We show first that $\sigma$ is a subgame $\phi^{\prime}$-maxmin strategy. For every $t \in \mathbb{N}$, there exists $t^{\prime} \geq t$ such that $\phi^{\prime}\left(c^{t^{\prime}}\right) \geq 1 /\left(t^{\prime}+1\right)$. Take the minimal $t^{\prime}$ with this property. We have that $u\left(\pi\left(\sigma, c^{t}\right)\right)=$ $u\left(c^{t^{\prime}} q\right)=t^{\prime} /\left(t^{\prime}+1\right)$. Because $\phi^{\prime}$ is a non-increasing function, we have that

$$
1-\phi^{\prime}\left(c^{t}\right) \leq 1-\phi^{\prime}\left(c^{t^{\prime}}\right) \leq 1-\frac{1}{t^{\prime}+1}=\frac{t^{\prime}}{t^{\prime}+1}=u\left(\pi\left(\sigma, c^{t}\right)\right) .
$$

We conclude that $\sigma$ is a subgame $\phi^{\prime}$-maxmin strategy. Because, for every $t \in \mathbb{N}, \phi^{\prime}\left(c^{t}\right) \leq \phi\left(c^{t}\right)$, the strategy $\sigma$ is a subgame $\phi$-maxmin strategy as well.

To identify subgame $\phi$-maxmin strategies, it is useful to define the function $\underline{u}: \mathcal{S}_{1} \times \mathcal{H} \rightarrow \mathbb{R}$ by

$$
\underline{u}(\sigma, h)=\inf _{\tau \in \mathcal{S}_{2}} \mathbb{E}_{h, \sigma, \tau}[u]
$$

The payoff $\underline{u}(\sigma, h)$ corresponds to the guarantee level that player 1 is expected to receive at history $h$ when playing the strategy $\sigma$. A strategy $\sigma \in \mathcal{S}_{1}$ is called a $\phi(h)$-maxmin strategy for the subgame at history $h$ if $\underline{u}(\sigma, h) \geq \underline{v}(h)-\phi(h)$.

For every strategy profile $(\sigma, \tau) \in \mathcal{S}_{1} \times \mathcal{S}_{2}$, for every $t \in \mathbb{N}$, define the stochastic variables $U_{\sigma, \tau}^{t}, \underline{U}_{\sigma}^{t}$, and $\underline{V}^{t}$ by letting $U_{\sigma, \tau}^{t}(p)=\mathbb{E}_{p_{\mid t}, \sigma, \tau}[u], \underline{U}_{\sigma}^{t}(p)=\underline{u}\left(\sigma, p_{\mid t}\right)$, and $\underline{V}^{t}(p)=\underline{v}\left(p_{\mid t}\right)$, respectively, for each play $p \in \mathcal{P}$. All three stochastic variables are $\mathcal{F}^{t}$-measurable. We have $\underline{U}_{\sigma}^{t} \leq U_{\sigma, \tau}^{t}$ and $\underline{U}_{\sigma}^{t} \leq \underline{V}^{t}$ everywhere on $\mathcal{P}$.

The next lemma states the submartingale property of guarantee levels. It says that the guarantee level that player 1 can expect to receive increases over time.

Lemma 3.3. (Submartingale property of guarantee levels) Let a strategy profile $(\sigma, \tau) \in$ $\mathcal{S}_{1} \times \mathcal{S}_{2}, t \in \mathbb{N}$, and a history $h \in \mathcal{H}^{t}$ of length $t$ be given.

[1] It holds that $\underline{u}(\sigma, h) \leq \mathbb{E}_{h, \sigma, \tau}\left[\underline{U}_{\sigma}^{t+1}\right]$.

[2] The process $\left(\underline{U}_{\sigma}^{t+n}\right)_{n \in \mathbb{N}}$ is a $\mathbb{P}_{h, \sigma, \tau}$-submartingale.

Proof. Take $\delta>0$. Let $\tau^{\prime} \in \mathcal{S}_{2}$ be such that $\tau^{\prime}(h)=\tau(h)$ and for each $(a, b, x) \in \mathcal{Z}$ it holds that

$$
\mathbb{E}_{(h, a, b, x), \sigma, \tau^{\prime}}[u] \leq \underline{u}(\sigma,(h, a, b, x))+\delta .
$$


We have that

$$
\begin{aligned}
\underline{u}(\sigma, h) & \leq \mathbb{E}_{h, \sigma, \tau^{\prime}}[u] \\
& =\sum_{(a, b, x) \in \mathcal{Z}} \sigma(h)(a) \cdot \tau(h)(b) \cdot q(x \mid h, a, b) \cdot \mathbb{E}_{(h, a, b, x), \sigma, \tau^{\prime}}[u] \\
& \leq \sum_{(a, b, x) \in \mathcal{Z}} \sigma(h)(a) \cdot \tau(h)(b) \cdot q(x \mid h, a, b) \cdot(\underline{u}(\sigma,(h, a, b, x))+\delta) \\
& =\mathbb{E}_{h, \sigma, \tau}\left[\underline{U}_{\sigma}^{t+1}\right]+\delta .
\end{aligned}
$$

The first claim follows since $\delta>0$ is arbitrary.

The second claim follows by Lemma A.1 in Appendix A.

\section{Conditions for strategies to be subgame $\phi$-maxmin}

\section{1 n-Day maxmin strategies and equalizing strategies}

The goal of this section is to provide necessary and sufficient conditions for a strategy to be subgame $\phi$-maxmin and to provide a characterization of subgame maxmin strategies.

Definition 4.1. A strategy $\sigma \in \mathcal{S}_{1}$ is an $n$-day $\phi$-maxmin strategy in the game $\Gamma_{x_{0}}(u)$ if for every $t \in \mathbb{N}$, for every history $h \in \mathcal{H}^{t}$ of length $t$, and for every strategy $\tau \in \mathcal{S}_{2}$,

$$
\mathbb{E}_{h, \sigma, \tau}\left[\underline{V}^{t+n}\right] \geq \underline{v}(h)-\phi(h) .
$$

Definition 4.2. A strategy $\sigma \in \mathcal{S}_{1}$ is $\phi$-equalizing in the game $\Gamma_{x_{0}}(u)$ if for every $t \in \mathbb{N}$, for every history $h \in \mathcal{H}^{t}$ of length $t$, and for every strategy $\tau \in \mathcal{S}_{2}$,

$$
u \geq \limsup _{t \rightarrow \infty} \underline{V}^{t}-\phi(h), \quad \mathbb{P}_{h, \sigma, \tau^{-}} \text {almost surely. }
$$

When $\phi=0$, we use the terms $n$-day maxmin and equalizing to mean $n$-day 0 -maxmin and 0-equalizing, respectively.

The first definition is very intuitive. It says that player 1 should play in such a way that, on average, the lower value increases over time. The notion of 1-day maxmin strategies is particularly well known in dynamic programming and stochastic games, see Puterman (1994). A simple characterization of 1-day maxmin strategies is provided in following theorem.

Theorem 4.3. Consider a strategy $\sigma \in \mathcal{S}_{1}$ in the game $\Gamma_{x_{0}}(u)$. The following three conditions are equivalent:

1. For each $n \in \mathbb{N}, \sigma$ is an $n$-day maxmin strategy.

2. $\sigma$ is a 1-day maxmin strategy.

3. For each history $h \in \mathcal{H}^{t}$ of length $t$ and each strategy $\tau \in \mathcal{S}_{2}$, the process $\left(\underline{V}^{t+n}\right)_{n \in \mathbb{N}}$ is $a \mathbb{P}_{h, \sigma, \tau}$-submartingale.

Proof. That [1] implies [2] is obvious. That [2] implies [3] follows by Lemma A.1 in Appendix A. Finally, that [3] implies [1] follows by the optional sampling theorem. 


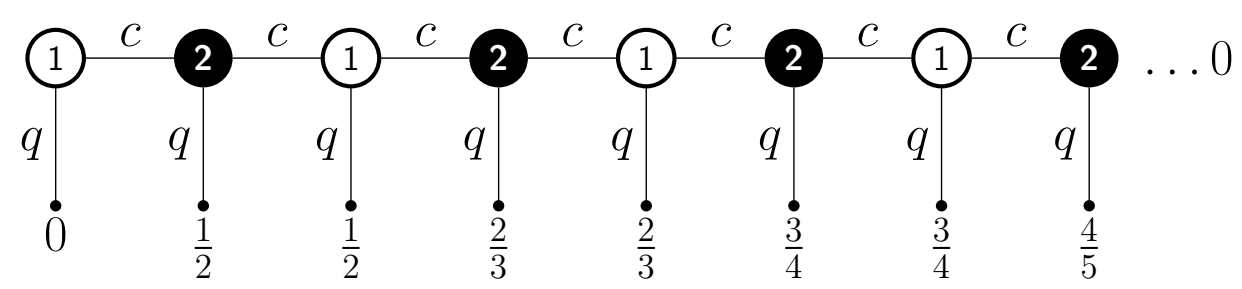

Figure 2: Strategies that are $n$-day maxmin may not be subgame maxmin.

A strategy is $\phi$-equalizing if, roughly speaking, it almost surely results in a play with an eventually good enough payoff, where eventually good enough means being about as large as the lower value in very deep subgames.

The following example illustrates both the notion of an $n$-day maxmin strategy and an equalizing strategy.

Example 4.4. Consider the centipede game depicted in Figure 2. At every history the active player can choose to continue $(c)$ or to quit $(q)$. As soon as a player decides to quit the game ends and in that case the payoff is as given in Figure 2. If the game continues indefinitely then the payoff is 0 . It is easily verified that, for every $t \in \mathbb{N}, \underline{v}\left(c^{2 t}\right)=\underline{v}\left(c^{2 t+1}\right)=(t+1) /(t+2)$.

In what follows we focus our attention on pure strategies and characterize the pure strategies that are $n$-day maxmin and the pure strategies that are equalizing.

Claim 1: A pure strategy $\sigma \in \mathcal{S}_{1}$ is an $n$-day maxmin strategy for every $n \in \mathbb{N}$ if and only if $\sigma\left(c^{2 t}\right)=c$ for every $t \in \mathbb{N}$.

Proof: If the active history is a history of player 1, i.e. $h=c^{2 t}$, and player 1 continues everywhere then for any strategy $\tau \in \mathcal{S}_{2}$ of the second player we have that $\mathbb{E}_{h, \sigma, \tau}\left[\underline{V}^{t+1}\right]=\underline{v}\left(c^{2 t+1}\right)$. If the active history is a history of player 2, i.e. $h=c^{2 t+1}$, then for any strategy $\tau \in \mathcal{S}_{2}$ of the second player we have that $\mathbb{E}_{h, \sigma, \tau}\left[\underline{V}^{t+1}\right]$ is either $u\left(c^{2 t+1} q\right)$ or $\underline{v}\left(c^{2 t+2}\right)$. Because in this game the lower value function is non-decreasing and $u\left(c^{2 t+1} q\right)=\underline{v}\left(c^{2 t}\right)$ we have that $\mathbb{E}_{h, \sigma, \tau}\left[\underline{V}^{t+1}\right] \geq \underline{v}(h)$ for every history $h \in \mathcal{H}$ and every strategy $\tau \in \mathcal{S}_{2}$. Hence $\sigma$ is a 1-day maxmin strategy. Using Theorem 4.3 we conclude that $\sigma$ is an $n$-day maxmin strategy for every $n \in \mathbb{N}$.

Conversely, assume there exists a history at which according to the strategy $\sigma$ player 1 quits. Let $c^{2 t}$ denote this history. Then we have for every $\tau \in \mathcal{S}_{2}$ that $\mathbb{E}_{c^{2 t}, \sigma, \tau}\left[\underline{V}^{t+1}\right]=$ $u\left(c^{2 t} q\right)<\underline{v}\left(c^{2 t}\right)$. We conclude that such a strategy $\sigma$ cannot be a 1-day maxmin strategy.

Claim 2: A pure strategy $\sigma \in \mathcal{S}_{1}$ is equalizing if and only if for infinitely many $t \in \mathbb{N}$ it holds that $\sigma\left(c^{2 t}\right)=q$.

Proof: If for infinitely many $t \in \mathbb{N}$ it holds that $\sigma\left(c^{2 t}\right)=q$, then for every strategy $\tau \in \mathcal{S}_{2}$ and every history $h \in \mathcal{H}$ there exists an $n \in \mathbb{N}$ such that the play $p$ generated from the history $h$ under the strategy profile $(\sigma, \tau)$ is $c^{n} q$. In this case it is clear that $\lim _{\sup } \rightarrow \infty \underline{v}\left(c^{n} q \mid t\right)=$ $u\left(c^{n} q\right)$, which proves that the strategy $\sigma$ is equalizing.

Conversely, assume $\sigma\left(c^{2 t}\right)=q$ for at most finitely many $t \in \mathbb{N}$. Then there exists a history after which player 1 always plays continue. Let $h$ denote this history and let $\tau \in \mathcal{S}_{2}$ denote the strategy of the second player in which he always continues. Then the play generated form the history $h$ under the strategy profile $(\sigma, \tau)$ is $c^{\infty}$. Observing that $0=u\left(c^{\infty}\right)<$ 
$\limsup _{t \rightarrow \infty} \underline{v}\left(c^{t}\right)=1$ concludes the proof that any strategy $\sigma$ in which player 1 quits at most finitely many times cannot be equalizing.

Consequence: In the centipede game depicted in Figure 2, player 1 does not have a pure strategy which is both $n$-day maxmin and equalizing.

The following theorem states sufficient conditions under which a strategy $\sigma$ of player 1 is a subgame $\phi$-maxmin strategy.

Theorem 4.5. (Sufficient condition) Let $\phi: \mathcal{H} \rightarrow[0, \infty)$ be a tolerance function. The strategy $\sigma \in \mathcal{S}_{1}$ is a subgame $\phi$-maxmin strategy in the game $\Gamma_{x_{0}}(u)$ if there exist tolerance functions $\phi_{1}: \mathcal{H} \rightarrow[0, \infty)$ and $\phi_{2}: \mathcal{H} \rightarrow[0, \infty)$ such that $\phi_{1}+\phi_{2} \leq \phi$ and

1. for every $n \in \mathbb{N}, \sigma$ is $n$-day $\phi_{1}$-maxmin,

2. $\sigma$ is $\phi_{2}$-equalizing.

Proof. Let $\phi_{1}, \phi_{2}$, and $\sigma$ be such that the conditions in the theorem are satisfied. We show that $\sigma$ is a subgame $\phi$-maxmin strategy.

Fix a history $h \in \mathcal{H}^{t}$ and a strategy $\tau \in \mathcal{S}_{2}$. Then we have that

$$
\begin{aligned}
\underline{v}(h)-\phi(h) & \leq \underline{v}(h)-\phi_{1}(h)-\phi_{2}(h) \\
& \leq \limsup _{n \rightarrow \infty} \mathbb{E}_{h, \sigma, \tau}\left[\underline{V}^{t+n}\right]-\phi_{2}(h) \\
& \leq \mathbb{E}_{h, \sigma, \tau}\left[\limsup _{n \rightarrow \infty} \underline{V}^{t+n}\right]-\phi_{2}(h) \\
& \leq \mathbb{E}_{h, \sigma, \tau}[u],
\end{aligned}
$$

where the second inequality holds since $\sigma$ is an $n$-day $\phi_{1}$-maxmin strategy, the third inequality is by Fatou lemma, and the last inequality holds since $\sigma$ is $\phi_{2}$-equalizing.

According to Theorem 4.5, to conclude that $\sigma$ is a subgame $\phi$-maxmin strategy, we should find tolerance functions $\phi_{1}$ and $\phi_{2}$ such that at each history their sum does not exceed $\phi$ and the strategy $\sigma$ is both $n$-day $\phi_{1}$-maxmin and $\phi_{2}$-equalizing.

Particularly natural are situations where the tolerance level does not increase as time progresses. More formally, the tolerance function $\phi$ is said to be non-increasing if $\phi(h) \geq \phi\left(h^{\prime}\right)$ whenever $h \prec h^{\prime}$. The following result states necessary conditions for a strategy to be subgame $\phi$-maxmin.

Theorem 4.6. (Necessary condition) Let $\sigma \in \mathcal{S}_{1}$ be a subgame $\phi$-maxmin strategy in the game $\Gamma_{x_{0}}(u)$. Then it holds that:

1. For every $n \in \mathbb{N}, \sigma$ is an $n$-day $\phi$-maxmin strategy.

2. If the tolerance function $\phi$ is non-increasing, then $\sigma$ is $\phi$-equalizing.

Proof. Let $\sigma \in \mathcal{S}_{1}$ be a subgame $\phi$-maxmin strategy in the game $\Gamma_{x_{0}}(u)$. Take a history $h \in \mathcal{H}^{t}$ of length $t$ and a strategy $\tau \in \mathcal{S}_{2}$.

We prove condition 1 . We have

$$
\mathbb{E}_{h, \sigma, \tau}\left[\underline{V}^{t+n}\right] \geq \mathbb{E}_{h, \sigma, \tau}\left[\underline{U}_{\sigma}^{t+n}\right] \geq \mathbb{E}_{h, \sigma, \tau}\left[\underline{U}_{\sigma}^{t}\right]=\underline{u}(\sigma, h) \geq \underline{v}(h)-\phi(h),
$$




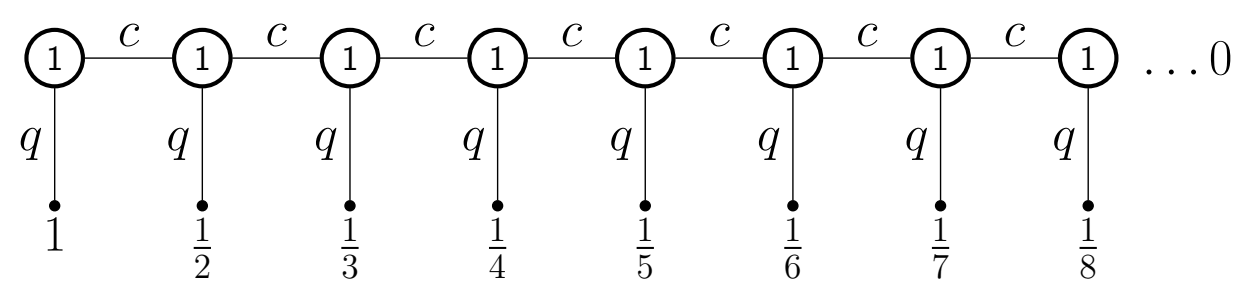

Figure 3: A 1-day $\phi$-maxmin strategy may not be $n$-day $\phi$-maxmin.

where the first inequality holds since for each play $p \in \mathcal{P}(h)$ we have $\underline{V}^{t+n}(p)=\underline{v}\left(p_{\mid t+n}\right) \geq$ $\underline{u}\left(\sigma, p_{\mid t+n}\right)=\underline{U}_{\sigma}^{t+n}(p)$. The second inequality holds by Lemma 3.3. The next equation holds since $t$ is the length of history $h$, and the final inequality holds since $\sigma$ is a subgame $\phi$-maxmin strategy.

We prove condition 2 . We have, $\mathbb{P}_{h, \sigma, \tau}$-almost surely,

$$
u(p)=\lim _{t \rightarrow \infty} \mathbb{E}_{p_{\mid t}, \sigma, \tau}[u] \geq \limsup _{t \rightarrow \infty}\left(\underline{v}\left(p_{\mid t}\right)-\phi\left(p_{\mid t}\right)\right) \geq \limsup _{t \rightarrow \infty} \underline{V}^{t}(p)-\phi(h),
$$

where the equality is by Levy's zero-one law (Lemma A.2 in Appendix A), the first inequality follows since $\sigma$ is a subgame $\phi$-maxmin strategy, and the second inequality holds since $\phi$ is non-increasing.

Notice that in the case of a non-zero tolerance function, the necessary and sufficient conditions do not coincide and we do not obtain a characterization of subgame $\phi$-maxmin strategies. We now turn to the case where the tolerance function $\phi$ is identically equal to 0 .

Corollary 4.7. A strategy $\sigma \in \mathcal{S}_{1}$ is a subgame maxmin strategy in the game $\Gamma_{x_{0}}(u)$ if and only if it is 1-day maxmin and equalizing.

When we compare the sufficient conditions of Theorem 4.5 to the sufficient conditions of Corollary 4.7, we notice that in the case of a non-zero tolerance function we require the strategy to be $n$-day $\phi$-maxmin. In the case of a zero tolerance function the corresponding sufficient conditions only require the strategy to be 1-day maxmin. The reason for this difference is that we should avoid that strategies accumulate the allowed tolerance levels, causing them to become too permissive over time. The following example illustrates this issue.

Example 4.8. Consider the decision problem depicted in Figure 3. Each period the decision maker can choose to continue $(c)$ or to quit $(q)$. Notice that $v\left(c^{t}\right)=1 /(t+1)$ and hence $\lim _{t \rightarrow \infty} v\left(c^{t}\right)=0$. Any strategy of player 1 is therefore equalizing. Furthermore, it is clear that in this decision problem the subgame maxmin strategy is unique and requires the decision maker to quit immediately. Now suppose the decision maker has the following tolerance function:

$$
\phi\left(c^{t}\right)=v\left(c^{t}\right)-v\left(c^{t+1}\right)=\frac{1}{t+1}-\frac{1}{t+2}, \quad t \in \mathbb{N} .
$$

Consider the strategy $\sigma$ where the decision maker always chooses to continue. From the definition of the tolerance function it follows that the strategy $\sigma$ is a 1-day $\phi$-maxmin 
strategy. Indeed, it holds that $v\left(c^{t+1}\right) \geq v\left(c^{t}\right)-\phi\left(c^{t}\right)$. It is also easily seen that the strategy $\sigma$ is equalizing. Nevertheless, it is clear that $\sigma$ is not a subgame $\phi$-maxmin strategy.

The underlying problem with the strategy $\sigma$ is that every time the decision maker chooses to continue this causes an acceptable loss in the value, but over time these losses add up to an unacceptable loss. The requirement that for every $n \in \mathbb{N}$ a strategy is $n$-day $\phi$-maxmin guarantees that the accumulated losses over any finite period of time remain acceptable.

If we require a strategy to be subgame maxmin, then we do not tolerate any losses. Therefore, the accumulation problem mentioned above will never occur and it will be sufficient to only require that the strategy is 1-day maxmin.

Example 4.9 is such that player 1 has a maxmin strategy in every subgame, but has no subgame maxmin strategy. From Corollary 4.7 it follows that any subgame maxmin strategy needs to be both 1-day maxmin and equalizing. The example presents a game where all the strategies are 1-day maxmin but none of them is equalizing and therefore subgame maxmin strategies do not exist.

Example 4.9. Consider the following perfect information game. Both players have two actions, left $(L)$ and right $(R)$, so $\mathcal{A}=\mathcal{B}=\{L, R\}$. The players take turns playing an action, which generates a play $p \in\{L, R\}^{\mathbb{N}}$. Let $r_{1}(p)$ and $r_{2}(p)$ denote the number of times that player 1 and player 2, respectively, play action $R$ during the play $p$ and define $r(p)=\min \left\{r_{1}(p), r_{2}(p)\right\}$. Player 1 obtains a payoff of 0 if both players choose $R$ infinitely often. When at least one of them chooses $R$ only a finite number of times, then player 1 receives a payoff of $r(p) /(r(p)+1)$. The payoff function $u$ is therefore obtained by defining, for $p \in\{L, R\}^{\mathbb{N}}$,

$$
u(p)= \begin{cases}\frac{r(p)}{r(p)+1}, & \text { if } r_{1}(p) \neq \infty \text { or } r_{2}(p) \neq \infty, \\ 0, & \text { if } r_{1}(p)=r_{2}(p)=\infty\end{cases}
$$

At each history $h \in \mathcal{H}$ the value of the game exists and is given by $v(h)=r_{2}(h) /\left(r_{2}(h)+1\right)$, where $r_{2}(h)$ denotes the number of times player 2 has chosen $R$ in the history $h$. Indeed, player 1 can guarantee this payoff by choosing the action $R \max \left\{r_{2}(h)-r_{1}(h), 0\right\}$ times after history $h$. Player 2 can guarantee to lose at most this amount by playing only left after history $h$. Hence at every history $h \in \mathcal{H}$ player 1 has an maxmin strategy.

For every history $h \in \mathcal{H}$ where player 1 takes an action and for every action $a \in \mathcal{A}$ we have that $r_{2}(h a)=r_{2}(h)$ and $v(h a)=v(h)$. Therefore, all strategies of player 1 are 1-day maxmin.

On the other hand, no equalizing strategy exists. To see this, take any strategy $\sigma \in \mathcal{S}_{1}$ for player 1 and consider the strategy $\tau \in \mathcal{S}_{2}$ in which player 2 always chooses $R$. Let $p$ be the play generated by the strategy profile $(\sigma, \tau)$. Then we have that $u(p)<1$ and $\lim _{t \rightarrow \infty} v\left(p_{\mid t}\right)=1$. It follows that $\sigma$ is not equalizing. Using Corollary 4.7 we conclude that player 1 does not have a subgame maxmin strategy.

Example 4.10. (Non-leavable decision problems) We consider a stochastic decision problem for player 1 , so player 2 is a dummy player. Let $r: \mathcal{X} \rightarrow \mathbb{R}$ be a bounded function that associates a reward to every state. The payoff function $u: \mathcal{P} \rightarrow \mathbb{R}$ is defined by

$$
u\left(x_{0}, a_{1}, x_{1}, a_{2}, \ldots\right)=\limsup _{t \rightarrow \infty} r\left(x_{t}\right), \quad\left(x_{0}, a_{1}, x_{1}, a_{2}, \ldots\right) \in \mathcal{P} .
$$


Maitra and Sudderth (1996) call decision problems with this structure non-leavable gambling problems and provide a characterization of optimal strategies in terms of thrifty and equalizing strategies. The 1-day maxmin strategies of Theorem 4.3 are the strategies that are thrifty after each history in Theorem 7.3 of Chapter 4 in Maitra and Sudderth (1996). Thus 1-day maxmin strategies can be seen as the "subgame" counterpart of thrifty strategies.

A strategy $\sigma$ is equalizing at a history $h \in \mathcal{H}$ if and only if for each $\epsilon>0$

$$
\left\{t \in \mathbb{N} \mid r\left(x_{t}\right) \geq \underline{v}\left(p_{\mid t}\right)-\epsilon\right\} \text { is infinite, } \mathbb{P}_{h, \sigma^{-}} \text {almost surely. }
$$

This follows from the fact that in a decision problem the process of lower values $\underline{V}^{t}$ is a $\mathbb{P}_{h, \sigma}$-supermartingale, and hence is a convergent sequence $\mathbb{P}_{h, \sigma}$-almost surely.

Using Theorem 7.7 of Chapter 4 in Maitra and Sudderth (1996), it follows that $\sigma$ is equalizing at $h$ according to their definition.

\subsection{The case of an upper semi-continuous payoff function}

The remainder of this section is devoted to the case where the payoff function is upper semicontinuous. We argue that in this case, any strategy of player 1 is equalizing. Because all upper semi-continuous functions are Borel measurable and because we assumed finite action sets and a countable state space, the value exists, see Maitra and Sudderth (1998) and Martin (1998), and the lower value equals the value.

The function $u$ is upper semi-continuous at a play $p \in \mathcal{P}$ if for every sequence $\left\{p_{t}\right\}_{t \in \mathbb{N}}$ of plays converging to $p$ it holds that

$$
\limsup _{t \rightarrow \infty} u\left(p_{t}\right) \leq u(p)
$$

Lemma 4.11. Let the payoff function $u$ be upper semi-continuous at the play $p$. Then we have that

$$
u(p) \geq \limsup _{t \rightarrow \infty} \underline{v}\left(p_{\mid t}\right) .
$$

Proof. Fix $\epsilon>0$. For every $t \in \mathbb{N}$, define $h_{t}=p_{\mid t}$ and let $p_{t} \in \mathcal{P}\left(h_{t}\right)$ be such that $u\left(p_{t}\right) \geq$ $\underline{v}\left(h_{t}\right)-\epsilon$. Such a play $p_{t}$ exists as player 1 can guarantee a payoff of at least $\underline{v}\left(h_{t}\right)-\epsilon$ at history $h_{t}$. Since the sequence $\left\{p_{t}\right\}_{t \in \mathbb{N}}$ converges to $p$, we have

$$
u(p) \geq \limsup _{t \rightarrow \infty} u\left(p_{t}\right) \geq \limsup _{t \rightarrow \infty} \underline{v}\left(h_{t}\right)-\epsilon .
$$

Since this holds for every $\epsilon>0$, the lemma follows.

In view of Lemma 4.11, we obtain the following corollary to Theorems 4.5, 4.6, and 4.7.

Corollary 4.12. Let $\Gamma_{x_{0}}(u)$ be such that $u$ is upper semi-continuous. Then each strategy of player 1 is equalizing. The strategy $\sigma \in \mathcal{S}_{1}$ is a subgame $\phi$-maxmin strategy if and only if for every $n \in \mathbb{N}$ it is an $n$-day $\phi$-maxmin strategy. The strategy $\sigma \in \mathcal{S}_{1}$ is a subgame maxmin strategy if and only if it is a 1-day maxmin strategy. 
Example 4.13. (Staying in the set game) Let some subset $\mathcal{X}^{*}$ of $\mathcal{X}$ be given. For a play $p=\left(x_{0}, a_{1}, b_{1}, x_{1}, a_{2}, b_{2} \ldots\right)$ we define $u(p)$ to be 1 if $x_{t} \in \mathcal{X}^{*}$ for every $t \in \mathbb{N}$ and to be 0 otherwise. Maitra and Sudderth (1996) refer to such a payoff function as "staying in a set" and in the computer science literature it goes under the name of "safety objective," see Bruyère (2017). Since $u$ is upper semi-continuous, any strategy $\sigma \in \mathcal{S}_{1}$ is equalizing.

Example 4.14. Consider again the centipede game depicted in Figure 2, but with one slight modification. If the game continues indefinitely, then player 1 receives a payoff of 2 instead of 0. The payoff function is now upper semi-continuous. As argued in Example 4.4, the strategy $\sigma$ in which player 1 continues at each history is 1-day maxmin. Because of Corollary 4.12, we can conclude that the strategy $\sigma$ is a subgame maxmin strategy.

\section{$5 \quad$ Existence of subgame $\phi$-maxmin strategies}

The goal of this section is to give sufficient conditions for the existence of a subgame $\phi$-maxmin strategy if the tolerance function $\phi$ is positive, so for every $h \in \mathcal{H}$ it holds that $\phi(h)>0$. We denote positive tolerance functions by $\phi>0$. These conditions are formally stated in Theorem 5.9.

The construction of the subgame $\phi$-maxmin strategy in Theorem 5.9 is as follows. Player 1 starts by playing a $\left(\phi\left(x_{0}\right) / 2\right)$-maxmin strategy. Next, at every history $h \in \mathcal{H}$ player 1 checks whether the strategy he is currently using is a $\phi(h)$-maxmin strategy for the subgame at history $h$. If this is the case he keeps using the strategy. If not, he switches to a $(\phi(h) / 2)$ maxmin strategy for the subgame at history $h$. We then use Theorem 4.5 to show that this construction leads to a subgame $\phi$-maxmin strategy. This type of construction is not new, and similar constructions were used for example in Rosenberg, Solan, and Vieille (2001), Solan and Vieille (2002), and Mashiah-Yaakovi (2015).

Fix a tolerance function $\phi>0$. For every history $h \in \mathcal{H}$, player 1 has a $(\phi(h) / 2)$-maxmin strategy for the subgame at history $h$, denoted by $\sigma^{h}$. The function $\psi: \mathcal{H} \rightarrow \mathcal{H}$ maps histories into histories and is such that player 1 is going to use strategy $\sigma^{\psi(h)}$ at subgame $h \in \mathcal{H}$. The function $\psi$ is used to describe when player 1 switches strategies and is recursively defined by setting $\psi\left(x_{0}\right)=x_{0}$ and, for every $h \in \mathcal{H}$, for every $z \in \mathcal{Z}$,

$$
\psi(h z)= \begin{cases}\psi(h), & \text { if } \underline{u}\left(\sigma^{\psi(h)}, h z\right) \geq \underline{v}(h z)-\phi(h z), \\ h z, & \text { otherwise. }\end{cases}
$$

The condition $\underline{u}\left(\sigma^{\psi(h)}, h z\right) \geq \underline{v}(h z)-\phi(h z)$ verifies whether the strategy to which player 1 switched last, $\sigma^{\psi(h)}$, is a $\phi(h z)$-maxmin strategy for the subgame at history $h z$. If this is the case, then there is no need to switch and $\psi(h z)=\psi(h)$. Otherwise, player 1 switches to $\sigma^{h z}$, which is achieved by setting $\psi(h z)=h z$. Formally, we define the switching strategy $\sigma^{\phi}: \mathcal{H} \rightarrow \Delta(\mathcal{A})$ by

$$
\sigma^{\phi}(h)=\sigma^{\psi(h)}(h), \quad h \in \mathcal{H} .
$$

The following example illustrates the construction of the switching strategy $\sigma^{\phi}$ and shows that it may not be subgame $\phi$-maxmin. 
Example 5.1. Consider again the centipede game depicted in Figure 2. We recall that, for every $t \in \mathbb{N}, \underline{v}\left(c^{2 t}\right)=\underline{v}\left(c^{2 t+1}\right)=(t+1) /(t+2)$. Take a tolerance function $\phi$ with the property that, for every $t \in \mathbb{N}, \phi\left(c^{2 t}\right)<1 /((t+1)(t+2))$.

Let $h \in \mathcal{H}$ be an active history for player 1 or player 2 and let $k \in \mathbb{N}$ be such that $h=c^{2 k}$ or $h=c^{2 k+1}$. The strategy $\sigma^{h}$ in which player 1 chooses continue at periods $0,2, \ldots, 2 k$ and chooses quit at every later period, i.e.

$$
\sigma^{h}\left(c^{2 t}\right)= \begin{cases}c, & \text { if } 2 t \leq 2 k \\ q, & \text { otherwise }\end{cases}
$$

is a maxmin strategy at subgame $h$.

We now consider the switching strategy $\sigma^{\phi}$. We show by induction that, for every $h \in H$, for every $z \in Z$, it holds that $\psi(h z)=h z$ if $h z$ is an active history of player 1 and $\psi(h z)=h$ if $h z$ is an active history of player 2 . The statement trivially holds for the initial history. Let $h$ be an active history of player 2 and let $t \in \mathbb{N}$ be such that $h=c^{2 t+1}$. Since $\sigma^{h}=\sigma^{c^{2 t}}$ is a maxmin strategy at subgame $h$, it holds that $\psi\left(c^{2 t+1}\right)=c^{2 t}$. Let $h$ be an active history of player 1 and let $t \in \mathbb{N} \backslash\{0\}$ be such that $h=c^{2 t}$. We have that

$$
\underline{u}\left(\sigma^{\psi\left(c^{2 t-1}\right)}, c^{2 t}\right)=\underline{u}\left(\sigma^{c^{2 t-2}}, c^{2 t}\right)=\frac{t}{t+1}=\underline{v}\left(c^{2 t}\right)-\frac{1}{(t+1)(t+2)}<\underline{v}\left(c^{2 t}\right)-\phi\left(c^{2 t}\right),
$$

so $\psi\left(c^{2 t}\right)=c^{2 t}$. Since the tolerance function $\phi$ is so stringent, it forces player 1 to switch at each of his active histories. For every $t \in \mathbb{N}$, it holds that $\sigma^{\phi}\left(c^{2 t}\right)=\sigma^{c^{2 t}}\left(c^{2 t}\right)=c$, so under $\sigma^{\phi}$ player 1 chooses $c$ at each of his active histories. The strategy $\sigma^{\phi}$ is not a subgame $\phi$-maxmin strategy as it fails to be $\phi$-equalizing, see Example 4.4.

Given the switching strategy $\sigma^{\phi}$, for every $k \in \mathbb{N}$ we define the strategy $\sigma^{k}: \mathcal{H} \rightarrow \Delta(\mathcal{A})$ such that it coincides with $\sigma^{\phi}$ as long as at most $k$ switches have been made and stops switching thereafter. Formally, we recursively define the function $\kappa: \mathcal{H} \rightarrow \mathbb{N}$ which counts the number of switches along a history $h$ by setting $\kappa\left(x_{0}\right)=0$ and, for all histories $h, h z \in \mathcal{H}$,

$$
\kappa(h z)= \begin{cases}\kappa(h), & \text { if } \underline{u}\left(\sigma^{\psi(h)}, h z\right) \geq \underline{v}(h z)-\phi(h z), \\ \kappa(h)+1, & \text { otherwise. }\end{cases}
$$

For every $k \in \mathbb{N}$, we define the stopping time $T_{k}: \mathcal{P} \rightarrow \mathbb{N} \cup\{\infty\}$ by

$$
T_{k}(p)=\inf \left\{t \in \mathbb{N} \mid \kappa\left(p_{\mid t}\right)=k\right\}, \quad p \in \mathcal{P} .
$$

The stopping time $T_{k}$ indicates the time at which switch $k$ occurred. Since, for $t<\infty$, the expression $T_{k}(p) \leq t$ only depends on the history up to period $t$, it holds that the set $\left\{p \in \mathcal{P} \mid T_{k}(p) \leq t\right\}$ is $\mathcal{F}^{t}$-measurable and $T_{k}$ is a stopping time indeed.

We now formally define $\sigma^{k}: \mathcal{H} \rightarrow \Delta(\mathcal{A})$. Take any $p \in \mathcal{P}(h)$ and let

$$
\sigma^{k}(h)= \begin{cases}\sigma^{\phi}(h), & \text { if } \kappa(h) \leq k, \\ \sigma^{h_{T_{k}(p)}(h),}, & \text { otherwise. }\end{cases}
$$

If $\kappa(h)>k$, then the time at which switch $k$ has occurred is the same for every $p \in \mathcal{P}(h)$, so it holds that $\sigma^{k}$ is well defined. 
For every $k \in \mathbb{N}$, let $\mathcal{R}_{k} \subseteq \mathcal{P}$ be the set of plays along which at least $k$ switches occur,

$$
\mathcal{R}_{k}=\left\{p \in \mathcal{P} \mid T_{k}(p)<\infty\right\},
$$

so $\mathcal{R}_{1} \supseteq \mathcal{R}_{2} \supseteq \mathcal{R}_{3} \supseteq \cdots$. Furthermore, let $\mathcal{R}_{\infty} \subseteq \mathcal{P}$ denote the set of plays along which infinitely many switches occur,

$$
\mathcal{R}_{\infty}=\cap_{k=1}^{\infty} \mathcal{R}_{k}
$$

The next result is very intuitive. Consider the strategies $\sigma^{k}, \sigma^{k+1}, \ldots$ All these strategies agree with $\sigma^{\phi}$ for as long as $\sigma^{\phi}$ does not require more than $k$ switches. Consequently, the measures that these strategies induce on $\mathcal{P}$ assign the same probability to any event that is "determined" before switch $k+1$ occurs, i.e. to any event in the sigma-algebra $\mathcal{F}^{T_{k+1}}$.

Lemma 5.2. Let a strategy $\tau \in \mathcal{S}_{2}$, a history $h \in \mathcal{H}$, and some $k \in \mathbb{N}$ be given. For $\sigma=\sigma^{k}, \sigma^{k+1}, \ldots, \sigma^{\phi}$, the probability measures $\mathbb{P}_{h, \sigma, \tau}$ all coincide on the sigma-algebra $\mathcal{F}^{T_{k+1}}$. Furthermore, these probability measures all agree on each universally measurable subset of $\mathcal{P} \backslash \mathcal{R}_{k+1}$.

Proof. A set $A$ of the universally measurable sigma-algebra $\mathcal{F}$ is called agreeable if for $\sigma=$ $\sigma^{k}, \sigma^{k+1}, \ldots, \sigma^{\phi}$ the measures $\mathbb{P}_{h, \sigma, \tau}$ all assign the same probability to $A$.

We argue first that each cylinder set in $\mathcal{F}^{T_{k+1}}$ is agreeable. A cylinder set $\mathcal{P}(h)$ is a member of $\mathcal{F}^{T_{k+1}}$ if and only if $\kappa(h) \leq k+1$. Let a cylinder set $\mathcal{P}\left(h^{\prime}\right)$ in $\mathcal{F}^{T_{k+1}}$ be given. Since $\kappa\left(h^{\prime}\right) \leq k+1$, we know that $\kappa\left(h^{\prime \prime}\right) \leq k$ for each history $h^{\prime \prime}$ preceding $h^{\prime}$. It follows that $\sigma^{k}\left(h^{\prime \prime}\right)=\sigma^{k+1}\left(h^{\prime \prime}\right)=\cdots=\sigma^{\phi}\left(h^{\prime \prime}\right)$. Since this applies to each history $h^{\prime \prime}$ that precedes $h^{\prime}$, the set $\mathcal{P}\left(h^{\prime}\right)$ is agreeable.

Now take any $E \in \mathcal{F}^{T_{k+1}}$. For $t \in \mathbb{N}$, let $E_{t}=E \cap\left\{p \in \mathcal{P} \mid T_{k+1}(p)=t\right\}$ and let $E_{\infty}=E \cap\left\{p \in \mathcal{P} \mid T_{k+1}(p)=\infty\right\}$. To show that $E$ is agreeable, it suffices to show that the sets $E_{t}$ and $E_{\infty}$ are.

Let some $t \in \mathbb{N}$ be given. We know that $E_{t}$ is a member of $\mathcal{F}^{t}$. Consequently, $E_{t}$ can be written as a disjoint union of cylinder sets in $\mathcal{F}^{t}$, say $E_{t}=\cup\left\{C_{n} \mid n \in \mathbb{N}\right\}$, with each $C_{n}$ a member of $\mathcal{F}^{t}$. Since each $C_{n}$ is a subset of the set $\left\{p \in \mathcal{P} \mid T_{k+1}(p)=t\right\}$, it is a member of $\mathcal{F}^{T_{k+1}}$, so is agreeable by the result of the second paragraph in the proof. It now follows that $E_{t}$ is agreeable.

To show that $E_{\infty}$ is agreeable, we make use of the fact that $E_{\infty}$ is a Borel set and of the regularity of $\sigma$ on the Borel sigma-algebra. Let $O$ be any open subset of $\mathcal{P}$ containing $E_{\infty}$. The set $O$ can be written as a disjoint union of cylinder sets, say $O=\cup\left\{\mathcal{P}\left(h_{n}\right) \mid n \in \mathbb{N}\right\}$. Without loss of generality it holds that, for every $n \in \mathbb{N}$ the set $\mathcal{P}\left(h_{n}\right)$ has a point in common with $E_{\infty}$. Thus in particular there is $p \in \mathcal{P}\left(h_{n}\right)$ with $T_{k+1}(p)=\infty$. This implies that $\kappa\left(h_{n}\right) \leq k$ and hence that $\mathcal{P}\left(h_{n}\right)$ is a member of $\mathcal{F}^{T_{k+1}}$. We conclude that each $\mathcal{P}\left(h_{n}\right)$ is agreeable by the result of the second paragraph in the proof. It follows that $O$ is an agreeable set.

To prove the second claim, we notice that all Borel subsets of $\mathcal{P} \backslash \mathcal{R}_{k+1}=\{p \in \mathcal{P} \mid$ $\left.T_{k+1}(p)=\infty\right\}$ are members of $\mathcal{F}^{T_{k+1}}$, so are agreeable. The result for universally measurable subsets of $\left\{p \in \mathcal{P} \mid T_{k+1}(p)=\infty\right\}$ follows since each such set differs from a Borel set by a negligible set. 
The following lemma is a special case of the optional sampling theorem with unbounded stopping times as presented in Yeh $\left(1995\right.$, p. 139). Assume we have specified an $\mathcal{F}^{\infty}$ measurable stochastic variable $\underline{U}_{\sigma}^{\infty}$. Let $T$ be a stopping time. We define the stochastic variable $\underline{U}_{\sigma}^{T}$ by letting it agree with $\underline{U}_{\sigma}^{t}$ on the set $\{p \in \mathcal{P}: T(p)=t\}$ for each $t \in \mathbb{N}$ and by letting it agree with $\underline{U}_{\sigma}^{\infty}$ on the set $\{p \in \mathcal{P}: T(p)=\infty\}$. The stochastic variable $\underline{U}_{\sigma}^{T}$ is then $\mathcal{F}^{T}$-measurable (Yeh, 1995, Theorem 3.28).

Exactly how the stochastic variable $\underline{U}_{\sigma}^{\infty}$ must be chosen is a rather subtle matter. In Lemmas 5.3, 5.5, and 5.6, $\underline{U}_{\sigma}^{\infty}$ is taken equal to some Borel measurable function that agrees with $u$ almost surely for the measure that is specified by the respective lemma. We cannot use $u$ itself, since $u$ is only assumed to be universally measurable. Neither can we fix $\underline{U}_{\sigma}^{\infty}$ in advance, because there is no function that would agree with $u$ almost surely with respect to all the measures that arise henceforth.

Lemma 5.3. (Optional sampling for guarantee level) Let a strategy profile $(\sigma, \tau) \in \mathcal{S}_{1} \times \mathcal{S}_{2}$, $t \in \mathbb{N}$, and a history $h \in \mathcal{H}^{t}$ of length $t$ be given. Let $\underline{U}_{\sigma}^{\infty}$ be an $\mathcal{F}^{\infty}$-measurable stochastic variable that $\mathbb{P}_{h, \sigma, \tau}$-almost surely coincides with $u$. Consider the stopping times $S$ and $T$ such that, for each $p \in \mathcal{P}(h), t \leq S(p) \leq T(p)$. Then

$$
\begin{aligned}
& \underline{U}_{\sigma}^{T} \leq \mathbb{E}_{h, \sigma, \tau}\left[u \mid \mathcal{F}^{T}\right], \quad \mathbb{P}_{h, \sigma, \tau} \text {-almost surely, } \\
& \underline{U}_{\sigma}^{S} \leq \mathbb{E}_{h, \sigma, \tau}\left[\underline{U}_{\sigma}^{T} \mid \mathcal{F}^{S}\right], \quad \mathbb{P}_{h, \sigma, \tau} \text {-almost surely. }
\end{aligned}
$$

Proof. The result follows by Theorem 8.16 in Yeh (1995, p. 139), applied to the process $\left(\underline{U}_{\sigma}^{n}\right)_{n \geq t}$ on the measurable space $\left(\mathcal{P}, \mathcal{F}, \mathbb{P}_{h, \sigma, \tau}\right)$ with a filtration $\left(\mathcal{F}^{n}\right)_{n \geq t}$.

We verify that the conditions of Theorem 8.16 in Yeh (1995) are satisfied. The process $\left(\underline{U}_{\sigma}^{n}\right)_{n \geq t}$ is a $\mathbb{P}_{h, \sigma, \tau}$-submartingale with respect to the filtration $\left(\mathcal{F}^{n}\right)_{n \geq t}$ by Lemma 3.3. Take $\xi$ of Theorem 8.16 in Yeh (1995) equal to $u$. Since $\underline{U}_{\sigma}^{\infty}$ is an $\mathcal{F}^{\infty}$-measurable stochastic variable that $\mathbb{P}_{h, \sigma, \tau}$-almost surely coincides with $u$, it is a version of $\mathbb{E}_{h, \sigma, \tau}\left[u \mid \mathcal{F}^{\infty}\right]$, as is required by the theorem.

Lastly, we verify that condition (1) of Theorem 8.16 in Yeh (1995) is satisfied. Notice that, for every $n \geq t$, for every play $p \in \mathcal{P}$, we have $\underline{U}_{\sigma}^{n}(p)=\underline{u}\left(\sigma, p_{\mid n}\right) \leq \mathbb{E}_{p_{\mid n}, \sigma, \tau}[u]$. The right-hand side of this inequality is a version of $\mathbb{E}_{h, \sigma, \tau}\left[u \mid \mathcal{F}^{n}\right]$. Consequently, $\underline{U}_{\sigma}^{n} \leq \mathbb{E}_{h, \sigma, \tau}\left[u \mid \mathcal{F}^{n}\right]$ holds $\mathbb{P}_{h, \sigma, \tau}$-almost surely, as desired.

The next lemma relates the guaranteed expected payoffs of strategies $\sigma^{k}$ and $\sigma^{k+1}$ at the moment of switch $k+1$. For this result, we choose $\underline{U}_{\sigma^{k}}^{\infty}=\underline{U}_{\sigma^{k+1}}^{\infty}$ to be any $\mathcal{F}^{\infty}$-measurable stochastic variable. How this stochastic variable is related to $u$ is unimportant. We write $\Phi^{t}$ to denote the $\mathcal{F}^{t}$-measurable stochastic variable given by $\Phi^{t}(p)=\phi\left(p_{\mid t}\right)$.

Lemma 5.4. Let $k \in \mathbb{N}$ and $\mathcal{F}^{\infty}$-measurable stochastic variables $\underline{U}_{\sigma^{k}}^{\infty}, \underline{U}_{\sigma^{k+1}}^{\infty}$ such that $\underline{U}_{\sigma^{k}}^{\infty}=$ $\underline{U}_{\sigma^{k+1}}^{\infty}$ be given. Then it holds that

$$
\underline{U}_{\sigma^{k}}^{T_{k+1}} \leq \underline{U}_{\sigma^{k+1}}^{T_{k+1}}-\frac{1}{2} \Phi^{T_{k+1}} \cdot I\left(T_{k+1}<\infty\right) .
$$

Proof. Let some $p \in \mathcal{P}$ be given. We distinguish the following two cases. 
CASE 1: $T_{k+1}(p)<\infty$. In this case at least $k+1$ switches occur along the play $p$. For $h=p_{\mid T_{k+1}(p)}$ we have the following inequalities

$$
\underline{u}\left(\sigma^{k}, h\right)<\underline{v}(h)-\phi(h)=\underline{v}(h)-\frac{1}{2} \phi(h)-\frac{1}{2} \phi(h) \leq \underline{u}\left(\sigma^{k+1}, h\right)-\frac{1}{2} \phi(h),
$$

where the first inequality holds since $\sigma^{k}$ is not a $\phi(h)$-maxmin strategy for the subgame at history $h$ and the second inequality holds because the strategy $\sigma^{k+1}$ is a $(\phi(h) / 2)$-maxmin strategy for the subgame at history $h$. Since $I\left(T_{k+1}(p)<\infty\right)=1$, (5.8) holds.

CASE 2: $T_{k+1}(p)=\infty$. In this case we have

$$
\underline{U}_{\sigma^{k}}^{T_{k+1}}(p)=\underline{U}_{\sigma^{k}}^{\infty}(p)=\underline{U}_{\sigma^{k+1}}^{\infty}(p)=\underline{U}_{\sigma^{k+1}}^{T_{k+1}}(p) .
$$

Thus (5.8) holds as an equality.

The following lemma states the intuitive property that for histories with less than $k+1$ switches or histories at which switch $k+1$ occurs, the strategy $\sigma^{k+1}$ guarantees at least the same payoff to player 1 than strategy $\sigma^{k}$.

Lemma 5.5. Let $t \in \mathbb{N}$ and a history $h \in \mathcal{H}^{t}$ of length $t$ be given. Let $k \in \mathbb{N}$ be such that $T_{k+1}(p) \geq t$ for every $p \in \mathcal{P}(h)$. Then it holds that $\underline{u}\left(\sigma^{k}, h\right) \leq \underline{u}\left(\sigma^{k+1}, h\right)$.

Proof. Fix strategy $\tau \in \mathcal{S}_{2}$ of player 2 .

We first define $\underline{U}_{\sigma^{k}}^{\infty}$. Consider the probability measure $\mathbb{Q}$ on the measurable space $(\mathcal{P}, \mathcal{F})$ given by $\mathbb{Q}(A)=\sum_{k \in \mathbb{N}} 2^{-k-1} \mathbb{P}_{h, \sigma^{k}, \tau}(A)$ for each $A \in \mathcal{F}$. Let $\bar{u}$ be an $\mathcal{F}^{\infty}$-measurable stochastic variable with the property that $\bar{u}=u, \mathbb{Q}$-almost surely. Since $\mathbb{P}_{h, \sigma^{k}, \tau}$ is absolutely continuous with respect to $\mathbb{Q}$ it holds that $\bar{u}=u, \mathbb{P}_{h, \sigma^{k}, \tau^{-}}$almost surely, for every $k \in \mathbb{N}$. We define $\underline{U}_{\sigma^{k}}^{\infty}$ to be equal to $\bar{u}$, for every $k \in \mathbb{N}$.

We now obtain the following inequalities. First, we have

$$
\underline{u}\left(\sigma^{k}, h\right) \leq \mathbb{E}_{h, \sigma^{k}, \tau}\left[\underline{U}_{\sigma^{k}}^{T_{k+1}}\right]
$$

an as instance of inequality (5.7) of Lemma 5.3 with $S=t$ and $T=T_{k+1}$. Secondly, from the fact that $\underline{U}_{\sigma^{k}}^{T_{k+1}}$ is an $\mathcal{F}^{T_{k+1}}$-measurable stochastic variable, we obtain by Lemma 5.2 that

$$
\mathbb{E}_{h, \sigma^{k}, \tau}\left[\underline{U}_{\sigma^{k}}^{T_{k+1}}\right]=\mathbb{E}_{h, \sigma^{k+1}, \tau}\left[\underline{U}_{\sigma^{k}}^{T_{k+1}}\right] .
$$

Thirdly, we know that

$$
\underline{U}_{\sigma^{k}}^{T_{k+1}} \leq \underline{U}_{\sigma^{k+1}}^{T_{k+1}} \leq \mathbb{E}_{h, \sigma^{k+1}, \tau}\left[u \mid \mathcal{F}^{T_{k+1}}\right], \quad \mathbb{P}_{h, \sigma^{k+1}, \tau^{-}} \text {almost surely, }
$$

where the first of these inequalities follows from Lemma 5.4 and the second one follows by inequality (5.6) of Lemma 5.3. Taking the expectation of the last array of inequalities with respect to $\mathbb{P}_{h, \sigma^{k+1}, \tau}$ and making use of the law of iterated expectation yields

$$
\mathbb{E}_{h, \sigma^{k+1}, \tau}\left[\underline{U}_{\sigma^{k}}^{T_{k+1}}\right] \leq \mathbb{E}_{h, \sigma^{k+1}, \tau}[u] .
$$

Combining these facts yields $\underline{u}\left(\sigma^{k}, h\right) \leq \mathbb{E}_{h, \sigma^{k+1}, \tau}[u]$. Taking the infimum over all strategies $\tau$ of player 2 completes the proof. 
Notice that a switch occurring at history $h$ increases the guarantee level of player 1 at $h$ by at least $\phi(h) / 2$. Although it is possible that player 1 switches infinitely many times along a play $p$, and therefore incurs infinitely many increases in his guarantee level along this play, the total overall increase in his guarantee level is bounded, since the payoff function itself is a bounded function. The next lemma provides the formal statement. We define

$$
M=\sup _{p \in \mathcal{P}}|u(p)|
$$

Lemma 5.6. Let $t \in \mathbb{N}$, a history $h \in \mathcal{H}^{t}$ of length $t$, and a strategy $\tau \in \mathcal{S}_{2}$ of player 2 be given. Then

$$
\sum_{k=\kappa(h)+1}^{\infty} \mathbb{E}_{h, \sigma^{\phi}, \tau}\left[\frac{1}{2} \Phi^{T_{k}} \cdot I\left(T_{k}<\infty\right)\right] \leq 2 M .
$$

Proof. As in the proof of Lemma 5.5, let $\bar{u}$ be any $\mathcal{F}^{\infty}$-measurable stochastic variable with

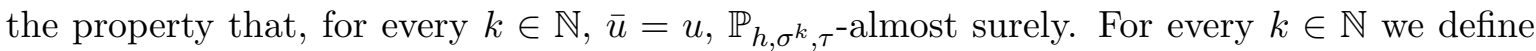
$\underline{U}_{\sigma^{k}}^{\infty}=\bar{u}$.

Let some $k>\kappa(h)$ be given. For every $p \in \mathcal{P}(h)$, it holds that $t \leq T_{k}(p) \leq T_{k+1}(p)$, so Lemma 5.3 implies

$$
\underline{U}_{\sigma^{k}}^{T_{k}} \leq \mathbb{E}_{h, \sigma^{k}, \tau}\left[\underline{U}_{\sigma^{k}}^{T_{k+1}} \mid \mathcal{F}^{T_{k}}\right], \quad \mathbb{P}_{h, \sigma^{k}, \tau^{-a l m o s t} \text { surely. }}
$$

We now have

$$
\mathbb{E}_{h, \sigma^{\phi}, \tau}\left[\underline{U}_{\sigma^{k}}^{T_{k}}\right]=\mathbb{E}_{h, \sigma^{k}, \tau}\left[\underline{U}_{\sigma^{k}}^{T_{k}}\right] \leq \mathbb{E}_{h, \sigma^{k}, \tau}\left[\underline{U}_{\sigma^{k}}^{T_{k+1}}\right]=\mathbb{E}_{h, \sigma^{\phi}, \tau}\left[\underline{U}_{\sigma^{k}}^{T_{k+1}}\right],
$$

where the two equalities follow from Lemma 5.2 and the fact that both $\underline{U}_{\sigma^{k}}^{T_{k}}$ and $\underline{U}_{\sigma^{k}}^{T_{k+1}}$ are $\mathcal{F}^{T_{k+1}}$-measurable stochastic variables, and the inequality follows by taking the expectation on both sides of inequality (5.10) and the law of iterated expectation.

Using Lemma 5.4 we can conclude that

$$
\begin{aligned}
\mathbb{E}_{h, \sigma^{\phi}, \tau}\left[\frac{1}{2} \Phi^{T_{k+1}} \cdot I\left(T_{k+1}<\infty\right)\right] & \leq \mathbb{E}_{h, \sigma^{\phi}, \tau}\left[\underline{U}_{\sigma^{k+1}}^{T_{k+1}}\right]-\mathbb{E}_{h, \sigma^{\phi}, \tau}\left[\underline{U}_{\sigma^{k}}^{T_{k+1}}\right] \\
& \leq \mathbb{E}_{h, \sigma^{\phi}, \tau}\left[\underline{U}_{\sigma^{k+1}}^{T_{k+1}}\right]-\mathbb{E}_{h, \sigma^{\phi}, \tau}\left[\underline{U}_{\sigma^{k}}^{T_{k}}\right] .
\end{aligned}
$$

Summing the preceding inequality over $k=\kappa(h)+1, \ldots, K$, we obtain

$$
\sum_{k=\kappa(h)+1}^{K} \mathbb{E}_{h, \sigma^{\phi}, \tau}\left[\frac{1}{2} \Phi^{T_{k}} \cdot I\left(T_{k}<\infty\right)\right] \leq \mathbb{E}_{h, \sigma^{\phi}, \tau}\left[\underline{U}_{\sigma^{K}}^{T_{K}}\right]-\mathbb{E}_{h, \sigma^{\phi}, \tau}\left[\underline{U}_{\sigma^{\kappa(h)+1}}^{T_{\kappa(h)+1}}\right] \leq 2 M .
$$

The result follows by taking the limit as $K \rightarrow \infty$.

The following lemma plays a crucial role in the proof of Theorem 5.9. Essentially it says that along almost any play $p \in \mathcal{P}$ only finitely many switches occur or the tolerance level goes to zero.

Lemma 5.7. For every history $h \in \mathcal{H}$, for every strategy $\tau \in \mathcal{S}_{2}$ of player 2 , it holds that

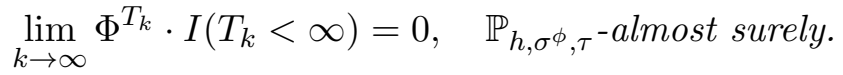


Proof. Let us write $X_{k}=\Phi^{T_{k}} \cdot I\left(T_{k}<\infty\right)$ and $k^{\prime}=\kappa(h)+1$. Since $X_{k} \geq 0$, the monotone convergence theorem implies that $\mathbb{E}_{h, \sigma^{\phi}, \tau}\left[\sum_{k=k^{\prime}}^{\infty} X_{k}\right]=\sum_{k=k^{\prime}}^{\infty} \mathbb{E}_{h, \sigma^{\phi}, \tau}\left[X_{k}\right]$. The latter expression is finite by Lemma 5.6. Thus $\sum_{k=k^{\prime}}^{\infty} X_{k}$ has a finite mean with respect to the probability measure $\mathbb{P}_{h, \sigma^{\phi}, \tau}$. Hence $\sum_{k=k^{\prime}}^{\infty} X_{k}<\infty$ holds $\mathbb{P}_{h, \sigma^{\phi}, \tau^{-}}$almost surely. This implies that $X_{k} \rightarrow 0$ holds $\mathbb{P}_{h, \sigma^{\phi}, \tau^{-}}$almost surely.

The following result is of interest in its own right. It states that the switching strategy $\sigma^{\phi}$ is $n$-day $\phi$-maxmin for every $n \in \mathbb{N}$.

Theorem 5.8. Let a game $\Gamma_{x_{0}}(u)$ and a tolerance function $\phi>0$ be given. For every $n \in \mathbb{N}$, the switching strategy $\sigma^{\phi}$ is n-day $\phi$-maxmin.

Proof. Let a history $h \in \mathcal{H}$ with length $t$ be given. Let $k=\kappa(h)$ denote the number of switches that has occurred along the history $h$. We obtain the following chain of inequalities

$$
\underline{v}(h)-\phi(h) \leq \underline{u}\left(\sigma^{k}, h\right) \leq \underline{u}\left(\sigma^{t+n}, h\right) \leq \mathbb{E}_{h, \sigma^{t+n}, \tau}\left[\underline{U}_{\sigma^{t+n}}^{t+n}\right] \leq \mathbb{E}_{h, \sigma^{t+n}, \tau}\left[\underline{V}^{t+n}\right]=\mathbb{E}_{h, \sigma^{\phi}, \tau}\left[\underline{V}^{t+n}\right],
$$

where the first inequality holds since $\sigma^{k}$ is a $\phi(h)$-maxmin strategy for the subgame at history $h$, the second inequality holds by Lemma 5.5 since $k \leq t \leq t+n$, the third inequality holds by Lemma 3.3, and the fourth one follows since $\underline{U}_{\sigma^{t+n}}^{t+n} \leq \underline{V}^{t+n}$. The final equality follows from the fact that the stochastic variable $\underline{V}^{t+n}$ is $\mathcal{F}^{t+n}$-measurable and the fact that the strategy $\sigma^{t+n}$ coincides with $\sigma^{\phi}$ at least until time $t+n$.

We are now in a position to state the main result of this section, which gives sufficient conditions for the existence of subgame $\phi$-maxmin strategies.

Theorem 5.9. Let a game $\Gamma_{x_{0}}(u)$ and a tolerance function $\phi>0$ be given such that for every $p \in \mathcal{P}$ at least one of the following two conditions holds:

1. (point of upper semicontinuity) The function $u$ is upper semi-continuous at $p$.

2. (positive limit inferior) $\lim \inf _{t \rightarrow \infty} \phi\left(p_{\mid t}\right)>0$.

Then there exists a subgame $\phi$-maxmin strategy in the game $\Gamma_{x_{0}}(u)$.

Proof. We define the tolerance function $\phi^{\prime}$ by

$$
\phi^{\prime}(h)=\frac{1}{2} \min \left\{\phi\left(h^{\prime}\right) \mid h^{\prime} \preceq h\right\}, \quad h \in \mathcal{H} .
$$

Thus $\phi^{\prime}$ is a non-increasing tolerance function with $\phi^{\prime} \leq \frac{1}{2} \phi$.

We show that $\sigma^{\phi^{\prime}}$ is $\phi^{\prime}$-equalizing. Since $2 \phi^{\prime} \leq \phi$ and $\sigma^{\phi^{\prime}}$ is an $n$-day $\phi^{\prime}$-maxmin strategy for every $n \in \mathbb{N}$ by Theorem 5.8, it then follows from Theorem 4.5 that $\sigma^{\phi^{\prime}}$ is a subgame $\phi$-maxmin strategy.

Let $\mathcal{U}$ denote the set of plays $p \in \mathcal{P}$ at which $u$ is upper semi-continuous and let $\mathcal{I}$ denote the set of plays $p \in \mathcal{P}$ such that $\liminf _{t \rightarrow \infty} \phi\left(p_{\mid t}\right)>0$. By the assumption of the theorem it holds that $\mathcal{P}=\mathcal{U} \cup \mathcal{I}$. By the definition of $\phi^{\prime}$, we have $\liminf _{t \rightarrow \infty} \phi^{\prime}\left(p_{\mid t}\right)>0$ for each $p \in \mathcal{I}$.

Let some $t \in \mathbb{N}$, a history $h \in \mathcal{H}^{t}$, and a strategy $\tau \in \mathcal{S}_{2}$ be given.

STEP 1: For every $p \in \mathcal{U}, u(p) \geq \lim \sup _{n \rightarrow \infty} \underline{V}^{n}(p)-\phi^{\prime}$.

This follows directly from Lemma 4.11 . 
For every $k \in \mathbb{N}$, we define $\mathcal{J}_{k}=\left\{p \in \mathcal{I} \cap\left(\mathcal{R}_{k} \backslash \mathcal{R}_{k+1}\right) \mid \lim _{n \rightarrow \infty} U_{\sigma^{k}, \tau}^{n}(p)=u(p)\right\}$ and $\mathcal{J}=\bigcup_{k \in \mathbb{N}} \mathcal{J}_{k}$.

STEP 2: For every $p \in \mathcal{J}, u(p) \geq \lim \sup _{n \rightarrow \infty} \underline{V}^{n}(p)-\phi^{\prime}$.

Let $k \in \mathbb{N}$ and $p \in \mathcal{J}_{k}$ be given. Exactly $k$ switches occur along the play $p$ and the last switch occurs at time $T_{k}(p)$. By our construction of $\sigma^{\phi^{\prime}}$, this means that for each time $n>T_{k}(p)$ the strategy $\sigma^{k}$ is a $\phi^{\prime}\left(p_{\mid n}\right)$-maxmin strategy for the subgame at history $p_{\mid n}$, so $\underline{u}\left(\sigma^{k}, p_{\mid n}\right) \geq \underline{v}\left(p_{\mid n}\right)-\phi^{\prime}\left(p_{\mid n}\right)$. Since $U_{\sigma^{k}, \tau}^{n}(p) \geq \underline{u}\left(\sigma^{k}, p_{\mid n}\right)$ and since for $n \geq t$ we have $\phi^{\prime}\left(p_{\mid n}\right) \leq \phi^{\prime}(h)$, we conclude that for $n \geq t$

$$
U_{\sigma^{k}, \tau}^{n}(p) \geq \underline{V}^{n}(p)-\phi^{\prime}(h) .
$$

Taking the limit as $n$ goes to infinity, and making use of the fact that $p \in \mathcal{J}_{k}$, we obtain

$$
u(p)=\lim _{n \rightarrow \infty} U_{\sigma^{k}, \tau}^{n}(p) \geq \limsup _{n \rightarrow \infty} \underline{V}^{n}(p)-\phi^{\prime}(h) .
$$

STEP 3: $\mathbb{P}_{h, \sigma^{\phi^{\prime}, \tau}}\left(\mathcal{I} \cap \mathcal{R}_{\infty}\right)=0$.

Recall that by Lemma 5.7, $\Phi^{T_{k}} \cdot I\left(T_{k}<\infty\right)$ converges to 0 as $k$ goes to infinity, $\mathbb{P}_{h, \sigma^{\phi^{\prime}}, \tau^{-}}$ almost surely. Also recall that $\mathcal{R}_{\infty}$ is the set of plays where infinitely many switches occur. Thus $I\left(T_{k}<\infty\right)$ is identically equal to 1 on $\mathcal{R}_{\infty}$. Furthermore, $\liminf _{k \rightarrow \infty} \Phi^{T_{k}}>0$ everywhere on $\mathcal{I}$. We conclude that $\Phi^{T_{k}} \cdot I\left(T_{k}<\infty\right)$ does not converge to zero on $\mathcal{I} \cap \mathcal{R}_{\infty}$ and the result follows.

STEP 4: $\mathbb{P}_{h, \sigma^{\phi^{\prime}}, \tau}(\mathcal{U} \cup \mathcal{J})=1$.

For every $k \in \mathbb{N}$, it holds by Levy's zero-one law (Lemma A.2 in Appendix A) that

$$
\mathbb{P}_{h, \sigma^{k}, \tau}\left(\mathcal{J}_{k}\right)=\mathbb{P}_{h, \sigma^{k}, \tau}\left(\mathcal{I} \cap\left(\mathcal{R}_{k} \backslash \mathcal{R}_{k+1}\right)\right) .
$$

Using Lemma 5.2 twice yields

$$
\mathbb{P}_{h, \sigma^{\phi^{\prime}, \tau}}\left(\mathcal{J}_{k}\right)=\mathbb{P}_{h, \sigma^{k}, \tau}\left(\mathcal{J}_{k}\right)=\mathbb{P}_{h, \sigma^{k}, \tau}\left(\mathcal{I} \cap\left(\mathcal{R}_{k} \backslash \mathcal{R}_{k+1}\right)\right)=\mathbb{P}_{h, \sigma^{\phi^{\prime}, \tau}}\left(\mathcal{I} \cap\left(\mathcal{R}_{k} \backslash \mathcal{R}_{k+1}\right)\right) .
$$

We now have

$$
\mathbb{P}_{h, \sigma^{\phi^{\prime}}, \tau}(\mathcal{J})=\mathbb{P}_{h, \sigma^{\phi^{\prime}, \tau}}\left(\mathcal{I} \backslash \mathcal{R}_{\infty}\right)=\mathbb{P}_{h, \sigma^{\phi^{\prime}, \tau}}(\mathcal{I}),
$$

where the last equality follows from Step 3. Finally, we obtain

$$
\mathbb{P}_{h, \sigma^{\phi^{\prime}}, \tau}(\mathcal{U} \cup \mathcal{J}) \geq \mathbb{P}_{h, \sigma^{\phi^{\prime}}, \tau}(\mathcal{U} \backslash \mathcal{I})+\mathbb{P}_{h, \sigma^{\phi^{\prime}, \tau}}(\mathcal{J})=\mathbb{P}_{h, \sigma^{\phi^{\prime}}, \tau}(\mathcal{U} \backslash \mathcal{I})+\mathbb{P}_{h, \sigma^{\phi^{\prime}}, \tau}(\mathcal{I})=1,
$$

where the last equality follows from the fact that the sets $\mathcal{U}$ and $\mathcal{I}$ cover $\mathcal{P}(h)$.

Theorem 5.9 generalizes Proposition 11 in Mashiah-Yaakovi (2015), where the existence of a subgame $\epsilon$-optimal strategy in a two-player zero-sum stochastic game with Borel measurable payoff functions is shown. The tolerance function $\phi$ there is given by $\phi(h)=\epsilon$ for every $h \in \mathcal{H}$, so for every play the tolerance function has a positive limit inferior. Theorem 5.9 yields the existence of a subgame $\epsilon$-maxmin strategy. Because the Borel measurability of the payoff function guarantees the existence of the value (Maitra and Sudderth, 1998, and Martin, 1998), this is equivalent to proving the existence of a subgame $\epsilon$-optimal strategy.

In Section 6, we argue that Theorem 5.9 also provides further insight into the main result of Laraki, Maitra, and Sudderth (2013). There the authors prove among other things that if the payoff function is bounded and upper semi-continuous, then the first player has a subgame optimal strategy. 


\section{$6 \quad$ Subgame maxmin strategies}

The goal of this section is to explore the relationship between the concept of a subgame maxmin strategy and that of a subgame $\phi$-maxmin strategy for $\phi>0$. The main result of this section is the following theorem.

Theorem 6.1. For every game $\Gamma_{x_{0}}(u)$ there exists a tolerance function $\phi^{*}>0$ such that the following statements are equivalent:

1. The game $\Gamma_{x_{0}}(u)$ has a subgame maxmin strategy.

2. The game $\Gamma_{x_{0}}(u)$ has a subgame $\phi^{*}$-maxmin strategy.

Because every subgame maxmin strategy is a subgame $\phi$-maxmin strategy, statement 1 clearly implies statement 2 . To prove the converse, we construct a tolerance function $\phi^{*}>0$ such that the tolerance levels decrease rapidly along each play. Then, provided that player 1 has a subgame $\phi^{*}$-maxmin strategy, we use Corollary 4.7 from Section 4 to construct a subgame maxmin strategy.

Theorem 6.1 has the following corollary.

Corollary 6.2. The following statements are equivalent:

1. The game $\Gamma_{x_{0}(u)}$ has a subgame maxmin strategy.

2. For every $\phi>0$, the game $\Gamma_{x_{0}}(u)$ has a subgame $\phi$-maxmin strategy.

We would like to make two remarks. First, as the payoff function $u$ need not be continuous, one cannot simply use a continuity argument to prove that statement 2 of Corollary 6.2 implies statement 1. Second, note that the existence of a subgame $\epsilon$-maxmin strategy for every $\epsilon>0$ is a weaker requirement than statements 1 and 2 of Corollary 6.2. Indeed, as Example 3.2 already illustrated, there exist games which admit a subgame $\epsilon$-maxmin strategy for every $\epsilon>0$ but do not admit a subgame maxmin strategy.

The special case where the payoff-function is upper semi-continuous deserves some additional attention. From Maitra and Sudderth (1998) and Martin (1998) it follows that every two-player zero-sum stochastic game with a countable state space, finite action sets, and a Borel measurable payoff function admits a value. Because every upper semi-continuous payoff function is Borel measurable, the existence of the value in our model is guaranteed. From Theorem 5.9 we obtain the existence of a subgame $\phi$-optimal strategy for every $\phi>0$. Combining this with Corollary 6.2 shows that player 1 has a subgame optimal strategy. Hereby we obtain a special case of the result by Laraki, Maitra, and Sudderth (2013), where the authors allow the state space to be a Borel subset of a Polish space and transition probabilities to be determined by a Borel transition function.

When we are interested in pure strategies that guarantee the maxmin levels, we can strengthen the result of Theorem 6.1. In the context of simultaneous move games, pure strategies are of course rather restrictive. Still, there are important classes of games, such as perfect information games, in which they are natural and play a prominent role.

Theorem 6.3. For every game $\Gamma_{x_{0}}(u)$ there exists a tolerance function $\phi^{*}>0$ such that the following statements are equivalent: 


\section{The pure strategy $\sigma \in \mathcal{S}_{1}$ is a subgame maxmin strategy.}

2. The pure strategy $\sigma \in \mathcal{S}_{1}$ is a subgame $\phi^{*}$-maxmin strategy.

This section is structured as follows. We start by proving Theorem 6.3, which is easier and helps us explain the main ideas. Then we turn to the proof of Theorem 6.1.

\subsection{The proof of Theorem 6.3}

In this subsection we prove Theorem 6.3. Since the statement of this theorem is about pure strategies, the proof is less technical and the intuition is more transparent.

We only need to prove that there is $\phi^{*}>0$ such that statement 2 of Theorem 6.3 implies statement 1 of Theorem 6.3. From now on, for any $t \in \mathbb{N}$, history $h \in \mathcal{H}^{t}$ with some final state $x$, and mixed actions $m_{1} \in \Delta(\mathcal{A})$ and $m_{2} \in \Delta(\mathcal{B})$, we denote the expectation of the lower value at the next stage by

$$
\mathbb{E}_{h, m_{1}, m_{2}}\left[\underline{V}^{t+1}\right]=\sum_{a \in \mathcal{A}} \sum_{b \in \mathcal{B}} \sum_{x^{\prime} \in \mathcal{X}} q\left(x^{\prime} \mid h, a, b\right) \cdot \underline{v}\left(h, a, b, x^{\prime}\right) .
$$

The proof consists of two steps.

STEP 1. Construction of $\phi^{*}>0$.

For every $t \in \mathbb{N}$, for every history $h \in \mathcal{H}^{t}$, there exists a number $d(h)>0$ such that for every action $a \in \mathcal{A}$ of player 1 either one of the following holds:

- Action $a$ guarantees that the lower value does not drop in expectation: for every $m_{2} \in$ $\Delta(\mathcal{B}), \mathbb{E}_{h, a, m_{2}}\left[\underline{V}^{t+1}\right] \geq \underline{v}(h)$.

- There exists a mixed action $m_{2} \in \Delta(\mathcal{B})$ for player 2 such that, if player 1 uses action $a$, the lower value drops in expectation by more than $d(h): \mathbb{E}_{h, a, m_{2}}\left[\underline{V}^{t+1}\right]<\underline{v}(h)-d(h)$.

This statement is true because the action set $\mathcal{A}$ of player 1 is finite. We define $\phi^{*}>0$ as follows. For every $t \in \mathbb{N}$, for every history $h \in \mathcal{H}^{t}$, let $\phi^{*}(h)=\min \left\{d(h), 2^{-t}\right\}$. The term $2^{-t}$ is included so that the tolerance levels tend to 0 along each play. For every $p \in \mathcal{P}$, it holds that $\lim _{t \rightarrow \infty} \phi^{*}\left(p_{\mid t}\right)=0$.

STEP 2. If the pure strategy $\sigma \in \mathcal{S}_{1}$ is a subgame $\phi^{*}$-maxmin strategy, then $\sigma$ is a subgame maxmin strategy.

Let the pure strategy $\sigma \in \mathcal{S}_{1}$ be subgame $\phi^{*}$-maxmin. We verify that $\sigma$ satisfies the conditions of Corollary 4.7.

First we show that $\sigma$ is 1 -day maxmin. Fix $t \in \mathbb{N}$ and $h \in \mathcal{H}^{t}$. Let $a=\sigma(h)$ denote the action that $\sigma$ recommends at $h$. Then, for every mixed action $m_{2} \in \Delta(\mathcal{B})$ and strategy $\tau \in \mathcal{S}_{2}$ with $m_{2}=\tau(h)$, we have

$$
\mathbb{E}_{h, a, m_{2}}\left[\underline{V}^{t+1}\right]=\mathbb{E}_{h, \sigma, \tau}\left[\underline{V}^{t+1}\right] \geq \underline{v}(h)-\phi^{*}(h) \geq \underline{v}(h)-d(h),
$$

where the first inequality follows from the fact that $\sigma$ is subgame $\phi^{*}$-maxmin and by condition 1 of Theorem 4.6, and the second inequality follows from the definition of $\phi^{*}(h)$. Therefore, by the choice of $d(h)$ in Step 1 , for every $m_{2} \in \Delta(\mathcal{B})$ it holds that

$$
\mathbb{E}_{h, a, m_{2}}\left[\underline{V}^{t+1}\right] \geq \underline{v}(h) .
$$


Hence, for every strategy $\tau \in \mathcal{S}_{2}$ it holds that

$$
\mathbb{E}_{h, \sigma, \tau}\left[\underline{V}^{t+1}\right]=\mathbb{E}_{h, a, \tau(h)}\left[\underline{V}^{t+1}\right] \geq \underline{v}(h) .
$$

Thus, $\sigma$ is 1-day maxmin.

We show that $\sigma$ is equalizing. Take some $\tau \in \mathcal{S}_{2}$. Because $\sigma$ is subgame $\phi^{*}$-maxmin, for every $p \in \mathcal{P}(h)$, for every $n \geq t$, we have

$$
U_{\sigma, \tau}^{n}(p)=\mathbb{E}_{p_{\mid n}, \sigma, \tau}[u] \geq \underline{v}\left(p_{\mid n}\right)-\phi^{*}\left(p_{\mid n}\right)=\underline{V}^{n}(p)-\Phi^{* n}(p),
$$

where $\Phi^{* n}(p)=\phi^{*}\left(p_{\mid n}\right)$. We conclude that

$$
\lim _{n \rightarrow \infty} U_{\sigma, \tau}^{n} \geq \limsup _{n \rightarrow \infty}\left(\underline{V}^{n}-\Phi^{* n}\right)=\limsup _{n \rightarrow \infty} \underline{V}^{n}-\lim _{n \rightarrow \infty} \Phi^{* n}=\limsup _{n \rightarrow \infty} \underline{V}^{n},
$$

where the last equality from the fact that for all $p \in \mathcal{P}$ we have $\lim _{n \rightarrow \infty} \phi^{*}\left(p_{\mid n}\right)=0$, so

$$
u=\lim _{n \rightarrow \infty} U_{\sigma, \tau}^{n} \geq \limsup _{n \rightarrow \infty} \underline{V}^{n}, \quad \mathbb{P}_{h, \sigma, \tau} \text {-almost surely, }
$$

where the equality follows from Lemma A.2 in Appendix A. We have shown that $\sigma$ is equalizing.

\subsection{The one-shot game $\Upsilon_{h}$}

To prove Theorem 6.1, we first analyze a one-shot zero-sum game in this subsection. For each history, the one-shot game is such that the payoff is given by the lower value at the next stage. In the next subsection, we use these one-shot games to construct the tolerance function $\phi^{*}>0$.

For some $t \in \mathbb{N}$, let $h \in \mathcal{H}^{t}$ be a history in the game $\Gamma_{x_{0}}(u)$. The one-shot zero-sum game $\Upsilon_{h}$ is played as follows. Player 1 chooses an action $a \in \mathcal{A}$ and player 2 simultaneously chooses an action $b \in \mathcal{B}$. Then, player 1 receives from player 2 the amount $\mathbb{E}_{h, a, b}\left[\underline{V}^{t+1}\right]$. As the action sets $\mathcal{A}$ and $\mathcal{B}$ are finite, the game $\Upsilon_{h}$ has a value, which we denote by $w(h)$. Furthermore, both players have optimal mixed actions in the game $\Upsilon_{h}$.

The following lemma states that the value $w(h)$ of the one-shot game $\Upsilon_{h}$ equals the lower value $\underline{v}(h)$ at the history $h$ in the original game $\Gamma_{x_{0}}(u)$.

Lemma 6.4. For every history $h \in \mathcal{H}$, we have $w(h)=\underline{v}(h)$.

Proof. The proof is by contradiction. Fix $t \in \mathbb{N}$ and a history $h \in \mathcal{H}^{t}$. Now suppose that $w(h) \neq \underline{v}(h)$. Then we have either $w(h)>\underline{v}(h)$ or $w(h)<\underline{v}(h)$.

CASE 1: $w(h)>\underline{v}(h)$.

Let $\delta=w(h)-\underline{v}(h)$. We derive a contradiction by showing that, in the subgame of $\Gamma_{x_{0}}(u)$ at history $h$, player 1 can guarantee an expected payoff of at least $\underline{v}(h)+\delta / 2$.

Let $m_{1} \in \Delta(\mathcal{A})$ be an optimal mixed action for player 1 in the one-shot game $\Upsilon_{h}$. Let $\sigma \in \mathcal{S}_{1}$ be such that $\sigma(h)=m_{1}$ and such that it induces a $(\delta / 2)$-maxmin strategy for the subgame at each history in period $t+1$, i.e., for every $h^{\prime} \in \mathcal{H}^{t+1}$, for every $\tau \in \mathcal{S}_{2}$,

$$
\mathbb{E}_{h^{\prime}, \sigma, \tau}[u] \geq \underline{v}\left(h^{\prime}\right)-\frac{\delta}{2} .
$$


Then, for every $\tau \in \mathcal{S}_{2}$, it holds that

$$
\begin{aligned}
\mathbb{E}_{h, \sigma, \tau}[u] & =\mathbb{E}_{h, \sigma, \tau}\left[U_{\sigma, \tau}^{t}\right]=\mathbb{E}_{h, \sigma, \tau}\left[U_{\sigma, \tau}^{t+1}\right] \\
& \geq \mathbb{E}_{h, \sigma, \tau}\left[\underline{V}^{t+1}\right]-\frac{\delta}{2}=\mathbb{E}_{h, m_{1}, \tau(h)}\left[\underline{V}^{t+1}\right]-\frac{\delta}{2} \geq w(h)-\frac{\delta}{2}=\underline{v}(h)+\frac{\delta}{2},
\end{aligned}
$$

where the second equality follows from the fact that $\left(U_{\sigma, \tau}^{n}\right)_{n \geq t}$ is a $\mathbb{P}_{h, \sigma, \tau}$-martingale, the first inequality follows from (6.2), and the second inequality follows from the choice of $m_{1}$.

CASE 2: $w(h)<\underline{v}(h){ }^{1}$

Let $\delta=\underline{v}(h)-w(h)$. We derive a contradiction by showing that, for every strategy of player 1 , there is a strategy for player 2 such that the expected payoff is at most $\underline{v}(h)-\delta / 2$ in the subgame of $\Gamma_{x_{0}}(u)$ at history $h$.

Fix $\sigma \in \mathcal{S}_{1}$ and let $m_{1}=\sigma(h)$. Let $m_{2} \in \Delta(\mathcal{B})$ be an optimal mixed action for player 2 in the one-shot game $\Upsilon_{h}$. Let $\tau \in \mathcal{S}_{2}$ be such that $\tau(h)=m_{2}$ and the expected payoff under $(\sigma, \tau)$ in the subgame at each history $h^{\prime}$ at period $t+1$ is at most the lower value $\underline{v}\left(h^{\prime}\right)+\delta / 2$, i.e., for every $h^{\prime} \in \mathcal{H}^{t+1}$,

$$
\mathbb{E}_{h^{\prime}, \sigma, \tau}[u] \leq \underline{v}\left(h^{\prime}\right)+\frac{\delta}{2} .
$$

We have that

$$
\begin{aligned}
\underline{v}(h)-\frac{\delta}{2} & =w(h)+\frac{\delta}{2} \geq \mathbb{E}_{h, m_{1}, m_{2}}\left[\underline{V}^{t+1}\right]+\frac{\delta}{2} \\
& \geq \mathbb{E}_{h, m_{1}, m_{2}}\left[U_{\sigma, \tau}^{t+1}\right]=\mathbb{E}_{h, \sigma, \tau}\left[U_{\sigma, \tau}^{t+1}\right]=\mathbb{E}_{h, \sigma, \tau}\left[U_{\sigma, \tau}^{t}\right]=\mathbb{E}_{h, \sigma, \tau}[u],
\end{aligned}
$$

where the first inequality follows from the choice of $m_{2}$, the second inequality follows from (6.3), and the penultimate equality follows from the fact that $\left(U_{\sigma, \tau}^{n}\right)_{n \geq t}$ is a $\mathbb{P}_{h, \sigma, \tau}$-martingale. Because $\sigma$ is chosen arbitrarily, we have derived a contradiction with the definition of the lower value $\underline{v}(h)$.

The total variation distance between two mixed actions $m_{1}, n_{1} \in \Delta(\mathcal{A})$ of player 1 is defined as

$$
\left\|m_{1}-n_{1}\right\|_{\mathrm{TV}}=\sum_{a \in \mathcal{A}}\left|m_{1}(a)-n_{1}(a)\right| .
$$

The total variation distance between two probability measures $\mathbb{P}$ and $\mathbb{P}^{\prime}$ on $(\mathcal{P}, \mathcal{F})$ is defined as

$$
\left\|\mathbb{P}-\mathbb{P}^{\prime}\right\|_{\mathrm{TV}}=\sup \left\{\sum_{i=1}^{n}\left|\mathbb{P}\left(F_{i}\right)-\mathbb{P}^{\prime}\left(F_{i}\right)\right|: F_{1}, \ldots, F_{n} \in \mathcal{F} \text { and }\left\{F_{1}, \ldots, F_{n}\right\} \text { is a partition of } \mathcal{P}\right\} .
$$

Let $t \in \mathbb{N}$ and a history $h \in \mathcal{H}^{t}$ be given. Let $O_{h} \subseteq \Delta(\mathcal{A})$ denote the set of optimal mixed actions of player 1 in the one-shot game $\Upsilon_{h}$. By Lemma 6.4 it holds that

$$
O_{h}=\left\{m_{1} \in \Delta(\mathcal{A}) \mid \text { for every } m_{2} \in \Delta(\mathcal{B}), \mathbb{E}_{h, m_{1}, m_{2}}\left[\underline{V}^{t+1}\right] \geq \underline{v}(h)\right\} .
$$

Note that $O_{h}$ is a compact subset of $\Delta(\mathcal{A})$. For every $m_{1} \in \Delta(\mathcal{A})$, the distance of $m_{1}$ to $O_{h}$ is defined by

$$
\left\|m_{1}-O_{h}\right\|_{\mathrm{TV}}=\min _{n_{1} \in O_{h}}\left\|m_{1}-n_{1}\right\|_{\mathrm{TV}}
$$

\footnotetext{
${ }^{1}$ The proof of this case in not symmetric to the proof of Case 1 , because we consider the lower value. Imitating the proof of Case 1 for player 2 would yield results in terms of the upper value.
} 
Due to the compactness of $O_{h}$, the minimum is attained. For $\delta>0$, let $D_{h}^{\delta}$ be the set of mixed actions of player 1 which have a distance of at least $\delta$ to the set $O_{h}$, so

$$
D_{h}^{\delta}=\left\{m_{1} \in \Delta(\mathcal{A}) \mid\left\|m_{1}-O_{h}\right\|_{\mathrm{TV}} \geq \delta\right\} .
$$

The mixed actions in $D_{h}^{\delta}$ are not optimal in the one-shot game $\Upsilon_{h}$. The following lemma says that the loss in utility caused by these mixed actions has a positive lower bound.

Lemma 6.5. Let $h \in \mathcal{H}$ and $\delta>0$ be given. If $D_{h}^{\delta}$ is non-empty, then there is $\epsilon>0$ such that for every $m_{1} \in D_{h}^{\delta}$ there exists $b \in \mathcal{B}$ such that

$$
\mathbb{E}_{h, m_{1}, b}\left[\underline{V}^{t+1}\right] \leq \underline{v}(h)-\epsilon .
$$

Proof. Assume $D_{h}^{\delta}$ is non-empty. Consider the function $e_{h}^{\delta}: D_{h}^{\delta} \rightarrow \mathbb{R}$ defined by

$$
e_{h}^{\delta}\left(m_{1}\right)=\underline{v}(h)-\min _{b \in \mathcal{B}} \mathbb{E}_{h, m_{1}, b}\left[\underline{V}^{t+1}\right], \quad m_{1} \in D_{h}^{\delta} .
$$

Since $\mathcal{B}$ is finite, the minimum exists. For every $m_{1} \in D_{h}^{\delta}$, we have $m_{1} \notin O_{h}$ and therefore there exists $m_{2} \in \Delta(\mathcal{B})$ such that $\mathbb{E}_{h, m_{1}, m_{2}}\left[\underline{V}^{t+1}\right]<\underline{v}(h)$. The function $e_{h}^{\delta}$ is therefore a positive and continuous function on a compact set, so has a positive minimum.

\subsection{Construction of the tolerance function $\phi^{*}$}

In this subsection, we define a positive tolerance function $\phi^{*}$. Fix a positive and decreasing sequence $\left(\delta_{t}\right)_{t \in \mathbb{N}}$ such that $\sum_{t=0}^{\infty} \delta_{t}<\infty$. Notice that this implies $\lim _{t \rightarrow \infty} \delta_{t}=0$.

For every $t \in \mathbb{N}$, for every history $h \in \mathcal{H}^{t}$, we define the constant $c(h)$ as follows. If the set $D_{h}^{\delta_{t}}$ is non-empty, then $c(h)$ is equal to the positive number $\epsilon$ of Lemma 6.5 and $c(h)=\delta_{t}$ otherwise. We define

$$
\phi^{*}(h)=\frac{\min \left\{c(h), \delta_{t}\right\}}{2} .
$$

Notice that $0<\phi^{*}(h)<\delta_{t}$.

We summarize the properties of the tolerance function $\phi^{*}$ :

1. For every history $h \in \mathcal{H}$, we have $\phi^{*}(h)>0$.

2. For every play $p \in \mathcal{P}$, we have $\sum_{t=0}^{\infty} \phi^{*}\left(p_{\mid t}\right) \leq \sum_{t=0}^{\infty} \delta_{t}<\infty$.

3. For every play $p \in \mathcal{P}, \lim _{t \rightarrow \infty} \phi^{*}\left(p_{\mid t}\right)=0$.

4. If the set $D_{h}^{\delta_{t}}$ is non-empty, then by the choice of $c(h)$, for every $m_{1} \in D_{h}^{\delta_{t}}$ there exists $b \in \mathcal{B}$ such that

$$
\mathbb{E}_{h, m_{1}, b}\left[\underline{V}^{t+1}\right] \leq \underline{v}(h)-c(h)<\underline{v}(h)-\phi^{*}(h) .
$$

The importance of $\sum_{t=0}^{\infty} \delta_{t}<\infty$ is underlined by the following lemma. Recall that $M=\sup _{p \in \mathcal{P}}|u(p)|$. 
Lemma 6.6. Let the strategies $\sigma, \sigma^{\prime} \in \mathcal{S}_{1}$ be such that, for every $t \in \mathbb{N}$, for every history $h \in \mathcal{H}^{t},\left\|\sigma(h)-\sigma^{\prime}(h)\right\|_{\mathrm{TV}} \leq \delta_{t}$. Then, for every strategy $\tau \in \mathcal{S}_{2}$, for every $t \in \mathbb{N}$, and for every history $h \in \mathcal{H}^{t}$,

$$
\left|\mathbb{E}_{h, \sigma, \tau}[u]-\mathbb{E}_{h, \sigma^{\prime}, \tau}[u]\right| \leq M \cdot \sum_{n=t}^{\infty} \delta_{n} .
$$

Proof. Let $\tau \in \mathcal{S}_{2}$ be given. It follows from a more general result in Abate, Redig, and Tkachev (2014, theorem 1) that, for every $t \in \mathbb{N}$, for every history $h \in \mathcal{H}^{t}$,

$$
\left\|\mathbb{P}_{h, \sigma, \tau}-\mathbb{P}_{h, \sigma^{\prime}, \tau}\right\|_{\mathrm{TV}} \leq \sum_{n=t}^{\infty} \delta_{n} .
$$

For completeness, we provide a direct proof of this inequality in Lemma B.3 in Appendix B. The claim of Lemma 6.6 follows directly.

Lemma 6.6 says the following. Consider two arbitrary strategies $\sigma, \sigma^{\prime} \in \mathcal{S}_{1}$ such that the total variation distance between the mixed actions at every history in period $t$ is at most $\delta_{t}$. Now consider $t \in \mathbb{N}$, a history $h \in \mathcal{H}^{t}$, and a strategy $\tau \in \mathcal{S}_{2}$ of player 2 . Then the expected payoffs under $(\sigma, \tau)$ and $\left(\sigma^{\prime}, \tau\right)$ in the subgame at $h$ differ at most the constant $M$ times $\sum_{n=t}^{\infty} \delta_{n}$. Note that this bound does not depend on the strategy $\tau$ and it only depends on the history $h$ through its period $t$. Moreover, these bounds tend to 0 as $t$ goes to infinity.

The importance of property 4 of the tolerance function is shown by the following lemma. It says that if $\sigma \in \mathcal{S}_{1}$ is a subgame $\phi^{*}$-maxmin strategy, then for every $h \in H$ the mixed action $\sigma(h)$ is close to the set of optimal mixed actions $O_{h}$ in the one-shot game $\Upsilon_{h}$.

Lemma 6.7. Let $\sigma \in \mathcal{S}_{1}$ be a subgame $\phi^{*}$-maxmin strategy. Then, for every $t \in \mathbb{N}$, for every history $h \in \mathcal{H}^{t}$, we have $\sigma(h) \notin D_{h}^{\delta_{t}}$, so $\left\|\sigma(h)-O_{h}\right\|_{\mathrm{TV}}<\delta_{t}$.

Proof. Because $\sigma$ is a subgame $\phi^{*}$-maxmin strategy, it follows from condition 1 of Theorem 4.6 that for every mixed action $m_{2} \in \Delta(\mathcal{B})$ of player 2

$$
\mathbb{E}_{h, \sigma(h), m_{2}}\left[\underline{V}^{t+1}\right] \geq \underline{v}(h)-\phi^{*}(h) .
$$

If $D_{h}^{\delta_{t}}$ is empty, then there is nothing to prove. If $D_{h}^{\delta_{t}}$ is non-empty, then property 4 of $\phi^{*}$ shows that $\sigma(h) \notin D_{h}^{\delta_{t}}$.

\subsection{The proof of Theorem 6.1}

Proof. Let $\Gamma_{x_{0}}(u)$ be a game and take the tolerance function $\phi^{*}$ as defined in Subsection 6.3. We only need to show that statement 2 implies statement 1 . Let $\sigma \in \mathcal{S}_{1}$ be a subgame $\phi^{*}$-maxmin strategy of $\Gamma_{x_{0}}(u)$.

With the help of $\sigma$, we define a strategy $\sigma^{*} \in \mathcal{S}_{1}$ in Step 1 of the proof. Then it is shown that $\sigma^{*}$ is a subgame maxmin strategy of $\Gamma_{x_{0}}(u)$ in Steps 2 and 3 of the proof by verifying that $\sigma^{*}$ satisfies the conditions of Corollary 4.7. 
STEP 1: Definition of $\sigma^{*} \in \mathcal{S}_{1}$.

Take $t \in \mathbb{N}$ and a history $h \in \mathcal{H}^{t}$. In view of Lemma 6.7, it holds that $\left\|\sigma(h)-O_{h}\right\|_{\mathrm{TV}}<\delta_{t}$. Therefore, there exists $m^{*}(h) \in O_{h}$ such that

$$
\left\|\sigma(h)-m^{*}(h)\right\|_{\mathrm{TV}}<\delta_{t}
$$

Now define $\sigma^{*}(h)=m^{*}(h)$.

STEP 2: $\sigma^{*}$ is 1-day maxmin.

Consider some $\tau \in \mathcal{S}_{2}$. For every $t \in \mathbb{N}$, for every $h \in \mathcal{H}^{t}$, since $\sigma^{*}(h) \in O_{h}$ we have that

$$
\mathbb{E}_{h, \sigma^{*}, \tau}\left[\underline{V}^{t+1}\right] \geq \underline{v}(h) .
$$

STEP 3: $\sigma^{*}$ is equalizing.

Consider some $\tau \in \mathcal{S}_{2}$. In view of (6.8) we can apply Lemma 6.6 to conclude that, for every $t \in \mathbb{N}$, for every $h \in \mathcal{H}^{t}$,

$$
\left|\mathbb{E}_{h, \sigma, \tau}[u]-\mathbb{E}_{h, \sigma^{*}, \tau}[u]\right| \leq M \cdot \sum_{n=t}^{\infty} \delta_{n} .
$$

Hence, for every $t \in \mathbb{N}$, for every history $h \in \mathcal{H}^{t}$, and for every $n \geq t$,

$$
\left|U_{\sigma, \tau}^{n}[u]-U_{\sigma^{*}, \tau}^{n}[u]\right| \leq M \cdot \sum_{i=n}^{\infty} \delta_{i}, \quad \mathbb{P}_{h, \sigma^{*}, \tau^{-}} \text {-almost surely. }
$$

Because $\sigma$ is subgame $\phi^{*}$-maxmin, for every history $h \in \mathcal{H}$, we have

$$
\mathbb{E}_{h, \sigma, \tau}[u] \geq \underline{v}(h)-\phi^{*}(h) .
$$

Thus, for every history $h \in \mathcal{H}$ it holds that

$$
u=\lim _{t \rightarrow \infty} U_{\sigma^{*}, \tau}^{t}=\lim _{t \rightarrow \infty} U_{\sigma, \tau}^{t} \geq \limsup _{t \rightarrow \infty}\left(\underline{V}^{t}-\Phi^{* t}\right)=\limsup _{t \rightarrow \infty} \underline{V}^{t}, \quad \mathbb{P}_{h, \sigma^{*}, \tau^{-} \text {almost surely }}
$$

where the first equality is due to Lemma A.2 in Appendix A, the second equality follows from (6.9), the inequality is by (6.10), and the last equality is a consequence of property 3 of $\phi^{*}$ from Subsection 6.3.

\section{Discussion}

In the previous sections we have assumed that action sets are finite and the state space is countable. The goal of this section is to analyze these assumptions further and to pinpoint where and how they were used.

Throughout this paper the restrictions on the cardinalities of action sets and state space were used to ensure the following properties:

1. The measurability of the lower value.

2. The existence of 1-day optimal actions in the game $\Upsilon_{h}$. 
The measurability of the lower value is crucial for the sufficient (Theorem 4.5) and necessary (Theorem 4.6) conditions of subgame $\phi$-maxmin strategies as well as for the characterization result (Corollary 4.7) for subgame maxmin strategies. Because the results in Sections 5 and 6 rely on this sufficient condition, the measurability of the lower value is indispensable throughout the paper. When working with infinite action sets and uncountable state spaces, Nowak (1985) and the references therein demonstrate that the (lower) value is not necessarily measurable.

Apart from ensuring that the lower value is measurable, the finiteness of action sets is used in Section 6 to guarantee the existence of 1-day optimal mixed actions in the game $\Upsilon_{h}$. Indeed, if action sets are finite, then for every history $h \in \mathcal{H}$ the game $\Upsilon_{h}$ is a finite zero-sum game and hence both players have optimal strategies.

\section{References}

[1] Abate, A., Redig, F., and Tkachev, I. (2014). On the effect of perturbation of conditional probabilities in total variation. Statistics and Probability Letters, 88, 1-8.

[2] Blackwell, D., Ferguson, T. (1968). The big match. Annals of Mathematical Statistics, 39, 159-163.

[3] Bogachev V.I. (2007). Measure Theory: Volume II, Springer-Verlag, Berlin Heidelberg.

[4] Bruyère, V. (2017). Computer aided synthesis: a game-theoretic approach. In Charlier, E., Leroy, J., and Rigo, M. (eds), Developments in Language Theory, Lecture Notes in Computer Science, 10396, Springer, pp. 3-35.

[5] Flesch, J., Predtetchinski, A. (2016). On refinements of subgame perfect $\epsilon$-equilibrium. International Journal of Game Theory, 45, 523-542.

[6] Flesch, J., Predtetchinski, A. and Sudderth, W. (2018). Characterization and simplification of optimal strategies in positive stochastic games. Forthcoming in Journal of Applied Probability.

[7] Flesch, J., Thuijsman, F., and Vrieze, O.J. (1998). Improving strategies in stochastic games. Proceedings of the 37th IEEE Conference on Decision and Control, Volume 3, IEEE, pp. 2674-2679.

[8] Fudenberg, D., Levine, D. (1983). Subgame-perfect equilibria of finite- and infinitehorizon games. Journal of Economic Theory, 31, 251-268.

[9] Gillette, D. (1957). Stochastic games with zero stop probabilities. In Dresher, M., Tucker, A.W., and Wolfe, P. (eds), Contributions to the Theory of Games, Vol III, Annals of Mathematics Studies, Volume 39, Princeton University Press, Princeton, New Jersey, pp. 179-187.

[10] Laraki, R., Maitra, A., and Sudderth, W. (2013). Two-person zero-sum stochastic games with semicontinuous payoff. Dynamic Games and Applications, 3, 162-171.

[11] Mailath, G., Postlewaite, A., and Samuelson, L. (2005). Contemporaneous perfect epsilon-equilibria. Games and Economic Behavior, 53, 126-140. 
[12] Maitra, A., Sudderth, W. (1996). Discrete Gambling and Stochastic Games, SpringerVerlag, New York.

[13] Maitra, A., Sudderth, W. (1998). Finitely additive stochastic games with Borel measurable payoffs. International Journal of Game Theory, 27, 257-267.

[14] Martin, D.A. (1998). The determinacy of Blackwell games. Journal of Symbolic Logic, $63,1565-1581$.

[15] Mashiah-Yaakovi, A. (2015). Correlated equilibria in stochastic games with Borel measurable payoffs. Dynamic Games and Applications, 5, 120-135.

[16] Nowak, A. (1985). Universally measurable strategies in zero-sum stochastic games. Annals of Probability, 13, 269-287.

[17] Puterman, M.L. (1994). Markov decision processes, discrete stochastic dynamic programming, John Wiley and Sons, Hoboken, New Jersey.

[18] Radner, R. (1980). Collusive behavior in noncooperative epsilon-equilibria of oligopolies with long but finite lives. Journal of Economic Theory, 22, 136-154.

[19] Rosenberg, D., Solan, E., and Vieille, N. (2001). Stopping games with randomized strategies. Probability Theory and Related Fields, 119, 433-451.

[20] Selten, R. (1965). Spieltheoretische Behandlung eines Oligopolmodells mit Nachfrageträgheit: Teil I: Bestimmung des dynamischen Preisgleichgewichts. Zeitschrift für die gesamte Staatswissenschaft, 121, 301-324.

[21] Solan, E., Vieille, N. (2002). Uniform value in recursive games. Annals of Applied Probability, 12, 1185-1201.

[22] Tversky, A., Kahneman, D. (1981). The framing of decisions and the psychology of choice. Science, 211, 453-458.

[23] Yeh, J., (1995). Martingales and Stochastic Analysis: Volume 1, World Scientific. 


\section{Appendix A: Universal measurability}

This appendix contains a review of the definitions of universally measurable sets, integrals of universally measurable functions, stopping times, and conditional expectations, as well as two technical lemmas that are used in the paper.

Universally measurable sets: Let $\left(\mathcal{P}, \mathcal{F}^{\infty}\right)$ be a measurable space, where $\mathcal{F}^{\infty}$ denotes the Borel sigma-algebra and let $\mathcal{M}$ denote the collection of all probability measures over this measurable space. For each probability measure $\mathbb{P} \in \mathcal{M}$, we can extend the probability space $\left(\mathcal{P}, \mathcal{F}^{\infty}, \mathbb{P}\right)$ to a complete probability space $\left(\mathcal{P}, \mathcal{F}_{\mathbb{P}}, \mathbb{P}^{\mathrm{c}}\right)$ by including all $\mathbb{P}$-negligible sets. To be more precise, let

$$
\mathcal{F}_{\mathbb{P}}^{0}=\left\{Q \subset \mathcal{P} \mid \exists Q^{\prime} \in \mathcal{F}^{\infty} \text { such that } \mathbb{P}\left(Q^{\prime}\right)=0 \text { and } Q \subseteq Q^{\prime}\right\}
$$

be the set of all subsets of $\mathbb{P}$-negligible sets of $\mathcal{F}^{\infty}$. We define

$$
\mathcal{F}_{\mathbb{P}}=\left\{Q \cup Q^{0} \subseteq \mathcal{P} \mid Q \in \mathcal{F}^{\infty} \text { and } Q^{0} \in \mathcal{F}_{\mathbb{P}}^{0}\right\}
$$

and we define $\mathbb{P}^{\mathrm{c}}: \mathcal{F}_{\mathbb{P}} \rightarrow[0,1]$ by

$$
\mathbb{P}^{\mathrm{c}}\left(Q \cup Q^{0}\right)=\mathbb{P}(Q), \quad Q \in \mathcal{F}^{\infty}, Q^{0} \in \mathcal{F}_{\mathbb{P}}^{0}
$$

It can be shown that $\left(\mathcal{P}, \mathcal{F}_{\mathbb{P}}, \mathbb{P}^{\mathrm{c}}\right)$ is a probability space. Now define

$$
\mathcal{F}=\bigcap_{\mathbb{P} \in \mathcal{M}} \mathcal{F}_{\mathbb{P}}
$$

It can be shown that $\mathcal{F}$ is a sigma-algebra that contains the Borel sigma-algebra $\mathcal{F}^{\infty}$ as a proper subset. The collection $\mathcal{F}$ is the universally measurable sigma-algebra and the elements of $\mathcal{F}$ are called universally measurable sets.

Integrals of universally measurable functions: A function $u: \mathcal{P} \rightarrow \mathbb{R}$ is called universally measurable if $u^{-1}[a, b] \in \mathcal{F}$ for every $[a, b] \subseteq \mathbb{R}$. The class of universally measurable functions contains the class of Borel measurable functions. Furthermore, for every universally measurable function there exists a Borel measurable function that coincides with it almost everywhere.

A function $g: \mathcal{P} \rightarrow \mathbb{R}$ is called a simple universally measurable function if it is of the form $g(p)=\sum_{i=1}^{n} c_{i} I\left(p \in Z_{i}\right)$, where $\left\{Z_{1}, \ldots, Z_{n}\right\}$ is a partition of $\mathcal{P}$ with $Z_{i} \in \mathcal{F}$ for all $i=1, \ldots, n$. The expected value of a simple universally measurable payoff with respect to a probability measure $\mathbb{P}$ is defined as

$$
\int_{p \in \mathcal{P}} g(p) \mathbb{P}(d p)=\sum_{i=1}^{n} c_{i} \mathbb{P}^{\mathrm{c}}\left(Z_{i}\right)
$$

Let $\mathcal{G}$ denote the set of simple universally measurable functions. The expected value of a bounded universally measurable function $u: \mathcal{P} \rightarrow \mathbb{R}$ is then given by

$$
\int_{p \in \mathcal{P}} u(p) \mathbb{P}(d p)=\sup _{\substack{g \in \mathcal{G} \\ g \leq u}} \int_{p \in \mathcal{P}} g(p) \mathbb{P}(d p)=\inf _{\substack{g \in \mathcal{G} \\ g \geq u}} \int_{p \in \mathcal{P}} g(p) \mathbb{P}(d p) .
$$


Stopping times: A stopping time is a function $T: \mathcal{P} \rightarrow \mathbb{N} \cup\{\infty\}$ such that for each $t \in \mathbb{N}$ the set $\{p \in \mathcal{P} \mid T(p)=t\}$ is an element of $\mathcal{F}^{t}$. Given a stopping time $T$, let $\mathcal{F}^{T}$ denote the sigma-algebra of sets $A \in \mathcal{F}^{\infty}$ such that $A \cap\{p \in \mathcal{P} \mid T(p)=t\} \in \mathcal{F}^{t}$ for each $t \in \mathbb{N}$.

For every $t \in \mathbb{N}$, let $X^{t}$ be an $\mathcal{F}^{t}$ measurable stochastic variable, and let $X^{\infty}$ be an $\mathcal{F}^{\infty}$ measurable stochastic variable. The stochastic variable $X^{T}$ is defined by letting it coincide with $X^{t}$ on $\{p \in \mathcal{P} \mid T(p)=t\}$ for each $t \in \mathbb{N}$ and with $X^{\infty}$ on $\{p \in \mathcal{P} \mid T(p)=\infty\}$. Following Yeh (1995, Theorem 3.28), it holds that $X^{T}$ is $\mathcal{F}^{T}$ measurable.

Conditional expectations: Consider a bounded stochastic variable $F: \mathcal{P} \rightarrow \mathbb{R}$, a strategy profile $(\sigma, \tau) \in \mathcal{S}_{1} \times \mathcal{S}_{2}$, and a history $h \in \mathcal{H}^{\ell}$ of length $\ell$. Let some $t \geq \ell$ be given. The conditional expectation of $F$ with respect to the sigma-algebra $\mathcal{F}^{t}$ and the measurable space $\left(\mathcal{P}, \mathcal{F}, \mathbb{P}_{h, \sigma, \tau}\right)$ is denoted by $\mathbb{E}_{h, \sigma, \tau}\left[F \mid \mathcal{F}^{t}\right]$. The conditional expectation $\mathbb{E}_{h, \sigma, \tau}\left[F \mid \mathcal{F}^{t}\right]$ can be identified with the stochastic variable $p \mapsto \mathbb{E}_{p_{\mid t}, \sigma, \tau}[F] .^{2}$

In what follows, we make repeatedly use of the following construction: Given a bounded function $f: \mathcal{H} \rightarrow \mathbb{R}$, we define for each $t \in \mathbb{N}$ the stochastic variable $F^{t}$ by letting $F^{t}(p)=$ $f\left(p_{\mid t}\right)$ for each play $p \in \mathcal{P}$. Notice that $F^{t}$ is $\mathcal{F}^{t}$ measurable.

Lemma A.1. Let $f: \mathcal{H} \rightarrow \mathbb{R}$ be a bounded function and let $(\sigma, \tau) \in \mathcal{S}_{1} \times \mathcal{S}_{2}$ be a strategy profile. The following two statements are equivalent:

[1] For each $t \in \mathbb{N}$ and each history $h \in \mathcal{H}^{t}$ of length $t$ it holds that $\mathbb{E}_{h, \sigma, \tau}\left[F^{t+1}\right] \geq f(h)$.

[2] For each each history $h \in \mathcal{H}^{\ell}$ of length $\ell$, the process $\left(F^{t}\right)_{t \geq \ell}$ is a $\mathbb{P}_{h, \sigma, \tau}$-submartingale with respect to the filtration $\left(\mathcal{F}^{t}\right)_{t \geq \ell}$ : for each $t \geq \ell$

$$
\mathbb{E}_{h, \sigma, \tau}\left[F^{t+1} \mid \mathcal{F}^{t}\right] \geq F^{t}, \quad \mathbb{P}_{h, \sigma, \tau} \text {-almost surely. }
$$

Proof. To see that [1] implies [2], take a history $h \in \mathcal{H}^{\ell}$ of length $\ell$ and time $t \geq \ell$. Take a play $p \in \mathcal{P}(h)$. Evaluating the left-hand side of (A.3) at $p$ yields $\mathbb{E}_{p_{\mid t}, \sigma, \tau}\left[F^{t+1}\right]$, which is at least $f\left(p_{\mid t}\right)=F^{t}(p)$ by condition [1].

To see that condition [2] implies [1] take a $t \in \mathbb{N}$ and history $h \in \mathcal{H}^{t}$. Take any play $p \in \mathcal{P}(h)$. The left-hand side of (A.3) is simply $\mathbb{E}_{h, \sigma, \tau}\left[F^{t+1}\right]$. The right-hand side of (A.3), evaluated at $p$, is $f(h)$. Condition [1] follows as $\mathbb{P}_{h, \sigma, \tau}$ is carried by the set $\mathcal{P}(h)$.

Lemma A.2 states a version of Levy's zero-one law for universally measurable functions. It relies on the fact that a universally measurable function can be approximated by a Borel measurable function.

Lemma A.2. (Levy's zero-one law for universally measurable functions) For every strategy profile $(\sigma, \tau) \in \mathcal{S}_{1} \times \mathcal{S}_{2}$, for every history $h \in \mathcal{H}$, we have

$$
\lim _{t \rightarrow \infty} U_{\sigma, \tau}^{t}=u, \quad \mathbb{P}_{h, \sigma, \tau} \text {-almost surely. }
$$

\footnotetext{
${ }^{2}$ As usual, a conditional expectation is not defined uniquely, since some histories might not be reached with positive probability under the strategy profile $(\sigma, \tau)$. Our particular choice is both convenient and inconsequential, since any two conditional probability systems coincide $\mathbb{P}_{h, \sigma, \tau}$-almost surely. We refer to Bogachev (2007, p. 350) for a careful discussion of conditional expectations.
} 
Proof. Fix a strategy profile $(\sigma, \tau) \in \mathcal{S}_{1} \times \mathcal{S}_{2}$ and a history $h \in \mathcal{H}$. Since $u$ is universally measurable, there exists a Borel measurable function $\bar{u}$ such that $u=\bar{u}, \mathbb{P}_{h, \sigma, \tau}$-almost surely. Then

$$
\lim _{t \rightarrow \infty} U_{\sigma, \tau}^{t}=\lim _{t \rightarrow \infty} \mathbb{E}_{h, \sigma, \tau}\left[u \mid \mathcal{F}^{t}\right]=\lim _{t \rightarrow \infty} \mathbb{E}_{h, \sigma, \tau}\left[\bar{u} \mid \mathcal{F}^{t}\right]=\mathbb{E}_{h, \sigma, \tau}\left[\bar{u} \mid \mathcal{F}^{\infty}\right]=\bar{u}
$$

$\mathbb{P}_{h, \sigma, \tau}$-almost surely. In the first equality we use the definition of the stochastic variable $U_{\sigma, \tau}^{t}$. The second equality follows from the fact that $u=\bar{u} \mathbb{P}_{h, \sigma, \tau}$-almost surely. The third equality follows from Levy's zero-one law (see e.g. Bogachev, 2007, Example 10.3.15). The last equality follows from the fact that $\bar{u}$ is $\mathcal{F}^{\infty}$ measurable. This is because $\bar{u}$ is Borel measurable and $\mathcal{F}^{\infty}$ is the Borel sigma-algebra on $\mathcal{P}$. The fact that $u=\bar{u} \mathbb{P}_{h, \sigma, \tau^{-}}$almost surely concludes the proof.

\section{Appendix B: the proof of inequality (6.7)}

The following statement follows from the more general result of Theorem 1 in Abate, Redig, and Tkachev (2014). For completeness, we provide a direct proof in this section.

Lemma B.3. Let $\sigma, \sigma^{\prime} \in \mathcal{S}_{1}$ be such that, for every $t \in \mathbb{N}$, for every $h \in \mathcal{H}^{t}, \| \sigma(h)-$ $\sigma^{\prime}(h) \|_{\mathrm{TV}} \leq \delta_{t}$. Then, for every strategy $\tau \in \mathcal{S}_{2}$, for every $t \in \mathbb{N}$, and for every history $h \in \mathcal{H}^{t}$,

$$
\left\|\mathbb{P}_{h, \sigma, \tau}-\mathbb{P}_{h, \sigma^{\prime}, \tau}\right\|_{\mathrm{TV}} \leq \sum_{i=t}^{\infty} \delta_{i} .
$$

The class $\mathcal{V} \subseteq \mathcal{F}$ is called an inner (outer) approximating class for the class $\mathcal{W} \subseteq \mathcal{F}$ if $\mathcal{V}$ is closed under unions (intersections) and if for every $\epsilon>0$, and for every probability measure $\mathbb{P} \in \mathcal{M}$ and every set $W \in \mathcal{W}$ there exists a set $V \in \mathcal{V}$ such that $V \subseteq W(V \supseteq W)$ and $|\mathbb{P}(W)-\mathbb{P}(V)| \leq \epsilon$.

Lemma B.4. If $\mathcal{V}$ is an inner (outer) approximating class for the class $\mathcal{W}$ and $\mathcal{V} \subseteq \mathcal{W}$, then it holds for every two probability measures $\mathbb{P} \in \mathcal{M}$ and $\mathbb{P}^{\prime} \in \mathcal{M}$ that

$$
\sup _{W \in \mathcal{W}}\left|\mathbb{P}(W)-\mathbb{P}^{\prime}(W)\right|=\sup _{V \in \mathcal{V}}\left|\mathbb{P}(V)-\mathbb{P}^{\prime}(V)\right| .
$$

Proof. Fix $\epsilon>0$. Assume that the class $\mathcal{V}$ is an inner approximating class for $\mathcal{W}$. The proof for an outer approximating class is similar. Fix two probability measures $\mathbb{P}$ and $\mathbb{P}^{\prime}$ and a set $W \in \mathcal{W}$. Then there exist sets $V \in \mathcal{V}$ and $V^{\prime} \in \mathcal{V}$ such that $V \subseteq W, V^{\prime} \subseteq W$, $|\mathbb{P}(W)-\mathbb{P}(V)| \leq \epsilon$, and $\left|\mathbb{P}^{\prime}(W)-\mathbb{P}^{\prime}\left(V^{\prime}\right)\right| \leq \epsilon$. Let $\tilde{V}=V \cup V^{\prime}$. Because $\mathcal{V}$ is closed under unions we have that $\tilde{V} \in \mathcal{V}$. Furthermore, it follows trivially that $\tilde{V} \subseteq W,|\mathbb{P}(W)-\mathbb{P}(\tilde{V})| \leq \epsilon$, and $\left|\mathbb{P}^{\prime}(W)-\mathbb{P}^{\prime}(\tilde{V})\right| \leq \epsilon$. We find that

$$
\begin{aligned}
\left|\mathbb{P}(W)-\mathbb{P}^{\prime}(W)\right| & =\left|\mathbb{P}(W)-\mathbb{P}(\tilde{V})+\mathbb{P}(\tilde{V})-\mathbb{P}^{\prime}(\tilde{V})+\mathbb{P}^{\prime}(\tilde{V})-\mathbb{P}^{\prime}(W)\right| \\
& \leq|\mathbb{P}(W)-\mathbb{P}(\tilde{V})|+\left|\mathbb{P}(\tilde{V})-\mathbb{P}^{\prime}(\tilde{V})\right|+\left|\mathbb{P}^{\prime}(\tilde{V})-\mathbb{P}^{\prime}(W)\right| \\
& \leq\left|\mathbb{P}(\tilde{V})-\mathbb{P}^{\prime}(\tilde{V})\right|+2 \epsilon .
\end{aligned}
$$


Because this holds for any $\epsilon>0$ and any set $W \in \mathcal{W}$, we can conclude that

$$
\sup _{W \in \mathcal{W}}\left|\mathbb{P}(W)-\mathbb{P}^{\prime}(W)\right| \leq \sup _{V \in \mathcal{V}}\left|\mathbb{P}(V)-\mathbb{P}^{\prime}(V)\right| .
$$

Because $\mathcal{V} \subseteq \mathcal{W}$ it is clear that:

$$
\sup _{W \in \mathcal{W}}\left|\mathbb{P}(W)-\mathbb{P}^{\prime}(W)\right| \geq \sup _{V \in \mathcal{V}}\left|\mathbb{P}(V)-\mathbb{P}^{\prime}(V)\right| .
$$

In the following lemma we use Lemma B.4 to simplify the computation of the total variation norm. Instead of having to compute the supremum over all sets of the universally measurable sigma-algebra it will be sufficient to compute the supremum over the subclass of open sets $\mathcal{O}^{t}, t \in \mathbb{N}$, defined by

$$
\mathcal{O}^{t}=\left\{\cup_{h \in H} \mathcal{P}(h) \mid H \subseteq \mathcal{H}^{t}\right\}
$$

The set $\mathcal{O}^{t}$ is the class of open sets such that all the plays sharing a common history at time $t$ are such that either all or none of them are contained in a specific open set.

Lemma B.5. For every $h \in \mathcal{H}$ it holds that

$$
\left\|\mathbb{P}_{h, \sigma, \tau}-\mathbb{P}_{h, \sigma^{\prime}, \tau}\right\|_{\mathrm{TV}}=\sup _{t \in \mathbb{N}, O \in \mathcal{O}^{t}}\left|\mathbb{P}_{h, \sigma, \tau}(O)-\mathbb{P}_{h, \sigma^{\prime}, \tau}(O)\right| .
$$

Proof. We define $\mathcal{O}=\cup_{t \in \mathbb{N}} \mathcal{O}^{t}$. Since any set in $\mathcal{O}$ is a union of open sets, it holds that $\mathcal{O}$ is a subset of the class of all open sets, $\mathcal{O}^{*}$. We now show that $\mathcal{O}$ is an inner approximating class for $\mathcal{O}^{*}$. Fix an open set $O^{*} \in \mathcal{O}^{*}$. For every $t \in \mathbb{N}$, we define $O^{t}=\left\{p \in \mathcal{P} \mid \mathcal{P}\left(p_{\mid t}\right) \subseteq O^{*}\right\}$. It is clear that for every $t \in \mathbb{N}$ we have $O^{t} \in \mathcal{O}$ and $O^{t} \subseteq O^{*}$. Furthermore, we have that $O^{1} \subseteq O^{2} \subseteq \ldots$ and $O^{*}=\cup_{t \in \mathbb{N}} O^{t}$. For every probability measure $\mathbb{P}$ it therefore holds that $\lim _{t \rightarrow \infty} \mathbb{P}\left(O^{t}\right)=\mathbb{P}\left(O^{*}\right)$, so for every $\epsilon>0$ there exists $t \in \mathbb{N}$ and $O^{t} \in \mathcal{O}^{t}$ such that $\left|\mathbb{P}\left(O^{*}\right)-\mathbb{P}\left(O^{t}\right)\right|<\epsilon$. Hence $\mathcal{O}$ is an inner approximating class of $\mathcal{O}^{*}$.

Because of the outer regularity of Borel measures on metric spaces, we have that the class of open sets is an outer approximating class for the class of Borel sets. In addition we have that the class of Borel sets is an inner approximating class for the class of universally measurable sets. Indeed, for any probability measure and any universally measurable set, there exists a Borel set which is contained in the universally measurable set and has the same probability. Repeated application of Lemma B.4 concludes the proof.

Proof of Lemma B.3: Fix $t \in \mathbb{N}$ and a history $h_{t} \in \mathcal{H}^{t}$. We prove by induction that for every $n \geq t$, for every $O \in \mathcal{O}^{n+1}$,

$$
\left|\mathbb{P}_{h_{t}, \sigma, \tau}(O)-\mathbb{P}_{h_{t}, \sigma^{\prime}, \tau}(O)\right| \leq \sum_{i=t}^{n} \delta_{i} .
$$

Induction basis $(n=t)$.

We prove that for every $O \in \mathcal{O}^{t+1},\left|\mathbb{P}_{h_{t}, \sigma, \tau}(O)-\mathbb{P}_{h_{t}, \sigma^{\prime}, \tau}(O)\right| \leq \delta_{t}$. Fix a set $O \in \mathcal{O}^{t+1}$. We define

$$
\mathcal{Z}_{h_{t}}=\left\{(a, b, x) \in \mathcal{A} \times \mathcal{B} \times \mathcal{X} \mid \mathcal{P}\left(h_{t} a b x\right) \subseteq O\right\} .
$$


Let $\mathcal{A}_{h_{t}}=\left\{a \in \mathcal{A} \mid \exists(b, x) \in \mathcal{B} \times \mathcal{X}:(a, b, x) \in \mathcal{Z}_{h_{t}}\right\}$ be the projection of the set $\mathcal{Z}_{h_{t}}$ on the set $\mathcal{A}$. Let $x_{t}$ denote the state at the history $h_{t}$. We have that

$$
\begin{aligned}
\mathbb{P}_{h_{t}, \sigma, \tau}(O) & =\sum_{(a, b, x) \in \mathcal{Z}_{h_{t}}} \sigma\left(h_{t}\right)(a) \cdot \tau\left(h_{t}\right)(b) \cdot q\left(x \mid a, b, x_{t}\right), \\
\mathbb{P}_{h_{t}, \sigma^{\prime}, \tau}(O) & =\sum_{(a, b, x) \in \mathcal{Z}_{h_{t}}} \sigma^{\prime}\left(h_{t}\right)(a) \cdot \tau\left(h_{t}\right)(b) \cdot q\left(x \mid a, b, x_{t}\right) .
\end{aligned}
$$

We find that

$$
\begin{aligned}
\left|\mathbb{P}_{h_{t}, \sigma, \tau}(O)-\mathbb{P}_{h_{t}, \sigma^{\prime}, \tau}(O)\right| & =\left|\sum_{(a, b, x) \in \mathcal{Z}_{h_{t}}}\left(\sigma\left(h_{t}\right)(a)-\sigma^{\prime}\left(h_{t}\right)(a)\right) \cdot \tau\left(h_{t}\right)(b) \cdot q\left(x \mid a, b, x_{t}\right)\right| \\
& \leq \sum_{(a, b, x) \in \mathcal{Z}_{h_{t}}}\left|\sigma\left(h_{t}\right)(a)-\sigma^{\prime}\left(h_{t}\right)(a)\right| \cdot \tau\left(h_{t}\right)(b) \cdot q\left(x \mid a, b, x_{t}\right) \\
& \leq \sum_{(a, b, x) \in \mathcal{A}_{h_{t}} \times \mathcal{B} \times \mathcal{X}}\left|\sigma\left(h_{t}\right)(a)-\sigma^{\prime}\left(h_{t}\right)(a)\right| \cdot \tau\left(h_{t}\right)(b) \cdot q\left(x \mid a, b, x_{t}\right) \\
& =\left[\sum_{a \in \mathcal{A}_{h_{t}}}\left|\sigma\left(h_{t}\right)(a)-\sigma^{\prime}\left(h_{t}\right)(a)\right|\right] \cdot\left[\sum_{(b, x) \in \mathcal{B} \times \mathcal{X}} \tau\left(h_{t}\right)(b) \cdot q\left(x \mid a, b, x_{t}\right)\right] \\
& \leq \sum_{a \in \mathcal{A}}\left|\sigma\left(h_{t}\right)(a)-\sigma^{\prime}\left(h_{t}\right)(a)\right|=\left\|\sigma\left(h_{t}\right)-\sigma^{\prime}\left(h_{t}\right)\right\|_{\mathrm{TV}} \leq \delta_{t} .
\end{aligned}
$$

\section{Induction step.}

From the induction hypotheses it follows that for every open $n$-level set $O^{n} \in \mathcal{O}^{n}$ with $n-1 \geq t$,

$$
\left|\mathbb{P}_{h_{t}, \sigma, \tau}\left(O^{n}\right)-\mathbb{P}_{h_{t}, \sigma^{\prime}, \tau}\left(O^{n}\right)\right| \leq \sum_{i=t}^{n-1} \delta_{i} .
$$

Fix a set $O \in \mathcal{O}^{n+1}$. We can assume without loss of generality that $\mathbb{P}_{h_{t}, \sigma, \tau}(O)-\mathbb{P}_{h_{t}, \sigma^{\prime}, \tau}(O) \geq 0$. Define

$$
\mathcal{H}_{+}^{n}=\left\{h_{n} \in \mathcal{H}^{n} \mid \mathbb{P}_{h_{t}, \sigma, \tau}\left(\mathcal{P}\left(h_{n}\right)\right)-\mathbb{P}_{h_{t}, \sigma^{\prime}, \tau}\left(\mathcal{P}\left(h_{n}\right)\right) \geq 0\right\} .
$$

We have that

$$
\begin{aligned}
& \mathbb{P}_{h_{t}, \sigma, \tau}(O)-\mathbb{P}_{h_{t}, \sigma^{\prime}, \tau}(O) \\
&= \sum_{h_{n} \in \mathcal{H}^{n}} \mathbb{P}_{h_{t}, \sigma, \tau}\left(O \mid \mathcal{P}\left(h_{n}\right)\right) \mathbb{P}_{h_{t}, \sigma, \tau}\left(\mathcal{P}\left(h_{n}\right)\right)-\sum_{h_{n} \in \mathcal{H}^{n}} \mathbb{P}_{h_{t}, \sigma^{\prime}, \tau}\left(O \mid \mathcal{P}\left(h_{n}\right)\right) \mathbb{P}_{h_{t}, \sigma^{\prime}, \tau}\left(\mathcal{P}\left(h_{n}\right)\right) \\
&= \sum_{h_{n} \in \mathcal{H}^{n}} \mathbb{P}_{h_{t}, \sigma, \tau}\left(O \mid \mathcal{P}\left(h_{n}\right)\right) \mathbb{P}_{h_{t}, \sigma, \tau}\left(\mathcal{P}\left(h_{n}\right)\right)-\sum_{h_{n} \in \mathcal{H}^{n}} \mathbb{P}_{h_{t}, \sigma, \tau}\left(O \mid \mathcal{P}\left(h_{n}\right)\right) \mathbb{P}_{h_{t}, \sigma^{\prime}, \tau}\left(\mathcal{P}\left(h_{n}\right)\right) \\
&+\sum_{h_{n} \in \mathcal{H}^{n}} \mathbb{P}_{h_{t}, \sigma, \tau}\left(O \mid \mathcal{P}\left(h_{n}\right)\right) \mathbb{P}_{h_{t}, \sigma^{\prime}, \tau}\left(\mathcal{P}\left(h_{n}\right)\right)-\sum_{h_{n} \in \mathcal{H}^{n}} \mathbb{P}_{h_{t}, \sigma^{\prime}, \tau}\left(O \mid \mathcal{P}\left(h_{n}\right)\right) \mathbb{P}_{h_{t}, \sigma^{\prime}, \tau}\left(\mathcal{P}\left(h_{n}\right)\right) \\
&= \sum_{h_{n} \in \mathcal{H}^{n}} \mathbb{P}_{h_{t}, \sigma, \tau}\left(O \mid \mathcal{P}\left(h_{n}\right)\right) \cdot\left(\mathbb{P}_{h_{t}, \sigma, \tau}\left(\mathcal{P}\left(h_{n}\right)\right)-\mathbb{P}_{h_{t}, \sigma^{\prime}, \tau}\left(\mathcal{P}\left(h_{n}\right)\right)\right) \\
&+\sum_{h_{n} \in \mathcal{H}^{n}} \mathbb{P}_{h_{t}, \sigma^{\prime}, \tau}\left(\mathcal{P}\left(h_{n}\right)\right) \cdot\left(\mathbb{P}_{h_{t}, \sigma, \tau}\left(O \mid \mathcal{P}\left(h_{n}\right)\right)-\mathbb{P}_{h_{t}, \sigma^{\prime}, \tau}\left(O \mid \mathcal{P}\left(h_{n}\right)\right)\right) \\
& \leq \sum_{h_{n} \in \mathcal{H}_{+}^{n}} \mathbb{P}_{h_{t}, \sigma, \tau}\left(O \mid \mathcal{P}\left(h_{n}\right)\right) \cdot\left(\mathbb{P}_{h_{t}, \sigma, \tau}\left(\mathcal{P}\left(h_{n}\right)\right)-\mathbb{P}_{h_{t}, \sigma^{\prime}, \tau}\left(\mathcal{P}\left(h_{n}\right)\right)\right) \\
&+\sum_{h_{n} \in \mathcal{H}^{n}} \mathbb{P}_{h_{t}, \sigma^{\prime}, \tau}\left(\mathcal{P}\left(h_{n}\right)\right) \cdot\left|\left(\mathbb{P}_{h_{t}, \sigma, \tau}\left(O \mid \mathcal{P}\left(h_{n}\right)\right)-\mathbb{P}_{h_{t}, \sigma^{\prime}, \tau}\left(O \mid \mathcal{P}\left(h_{n}\right)\right)\right)\right| \\
& \leq \sum_{h_{n} \in \mathcal{H}_{+}^{n}}\left(\mathbb{P}_{h_{t}, \sigma, \tau}\left(\mathcal{P}\left(h_{n}\right)\right)-\mathbb{P}_{h_{t}, \sigma^{\prime}, \tau}\left(\mathcal{P}\left(h_{n}\right)\right)\right)+\sum_{h_{n} \in \mathcal{H}^{n}} \mathbb{P}_{h_{t}, \sigma^{\prime}, \tau}\left(\mathcal{P}\left(h_{n}\right)\right) \cdot \delta_{n} \\
& \leq\left|\mathbb{P}_{h_{t}, \sigma, \tau}\left(\cup_{h_{n} \in \mathcal{H}_{+}^{n}} \mathcal{P}\left(h_{n}\right)\right)-\mathbb{P}_{h_{t}, \sigma^{\prime}, \tau}\left(\cup_{h_{n} \in \mathcal{H}_{+}^{n}} \mathcal{P}\left(h_{n}\right)\right)\right|+\delta_{n} \\
& \leq \sum_{i=t}^{n-1} \delta_{i}+\delta_{n}=\sum_{i=t}^{n} \delta_{i},
\end{aligned}
$$

where the fact that $\left|\left(\mathbb{P}_{h_{t}, \sigma, \tau}\left(O \mid \mathcal{P}\left(h_{n}\right)\right)-\mathbb{P}_{h_{t}, \sigma^{\prime}, \tau}\left(O \mid \mathcal{P}\left(h_{n}\right)\right)\right)\right| \leq \delta_{n}$ follows by assumption.

Using Lemma B.5 we can conclude that

$$
\begin{aligned}
\left\|\mathbb{P}_{h_{t}, \sigma, \tau}-\mathbb{P}_{h_{t}, \sigma^{\prime}, \tau}\right\|_{\mathrm{TV}} & =\sup _{n \in \mathbb{N}, O \in \mathcal{O}^{n}}\left|\mathbb{P}_{h_{t}, \sigma, \tau}(O)-\mathbb{P}_{h_{t}, \sigma^{\prime}, \tau}(O)\right| \\
& =\sup _{n \geq t, O \in \mathcal{O}^{n+1}}\left|\mathbb{P}_{h_{t}, \sigma, \tau}(O)-\mathbb{P}_{h_{t}, \sigma^{\prime}, \tau}(O)\right| \\
& \leq \sup _{n \geq t} \sum_{i=t}^{n} \delta_{i}=\sum_{i=t}^{\infty} \delta_{i}
\end{aligned}
$$

